\title{
12. RADIATION AND STRUCTURE OF THE SOLAR ATMOSPHERE (RADIATION ET STRUCTURE DE L'ATMOSPHÈRE SOLAIRE)
}

\author{
PRESIDENT: R. G. Giovanelli. \\ VICE-PRESIDENT: M. K. V. Bappu. \\ ORGANIZING COMMITTEE: R. G. Athay, J. M. Beckers, P. Delache, C. Jordan, G. M. Nikolsky, \\ E. H. Schröter.
}

\section{THE PHOTOSPHERE \\ (P. Mein)}

\section{A. Introduction}

Many investigations have been devoted to the photosphere during the last three years. Spatial and time resolution have experienced an improvement and the resolved structures on the solar surface have been studied extensively from an observational and theoretical point of view. At the same time, the importance of large scale structure and pole-equator effects for the understanding of the solar interior and active phenomena have been pointed out in several investigations. Line formation and non-LTE problems in inhomogeneous media are not yet solved, and extensive work in this area may be expected in the future. As far as the mean temperature model atmosphere is concerned, our knowledge has been improved by many investigations attempting to get an agreement between various observational techniques.

\section{B. Homogeneous Models}

The transition region between the convection zone and the photosphere was studied by Spruit (1974), using a mixing length formalism. The results match the HSRA model and an interior model. Allen et al. (1973) measured the limb darkening of infra-red continuum $(1 \mu-5 \mu)$ in order to test the temperature in the low layers of the HSRA model. The homogeneous model photosphere has been discussed most often for the case of the upper layers, probably because most IR and UV measurements are relevant to these heights. Fortunately the inhomogeneities are not too strong in the region lying between the penetrating convection and the chromosphere temperature rise.

Infra-red observations have been carried out in the range extending from $100 \mu$ to $1000 \mu$ by Stettler et al. $(1972,1975)$ and Gezari et al. (1973). The agreement with the HSRA model is good in the region of the temperature rise $(\lambda>300 \mu)$. Some dispersion occurs near the temperature minimum.

In the UV range, detailed observations have been carried out at short wavelengths (1200-2000 \&) by Brueckner et al. (1973), Nishi (1973, 1975), Ackerman et al. (1973). Samain et al. (1975) found the temperature minimum equal to $4430 \mathrm{~K}$ around $1580 \AA$. The solar fluxes between 2000 and $2700 \AA$ have also been observed by Simon (1974). Jordan et al. (1974) pointed out that the assumption of an inhomogeneous atmosphere is necessary to account for low brightness temperature values extending beyond the limb. The question of missing opacity sources continues to be an open question for the wavelength range $\lambda>1700 \AA$. Krishnaswamy et al. (1974) suggested a mechanism involving departure from LTE for $\mathrm{H}_{2}^{+}$. Other possibilities, such as negative ions, two-photon processes, forbidden photodissociations, have been investigated unsuccessfully by Landi degl'Innocenti et al. (1973). Anyway, better abundances ( $\mathrm{Fe}$ ) and new calculations of photoionisation cross sections would be required.

Blanketing effects have been studied by Kosak et al. $(1972,1973,1974)$. Assuming a radiative-convective equilibrium with mixing-length to scale height ratio of 2 , solar abundances from Withbroe (1971) and a $2 \mathrm{~km} \mathrm{~s}^{-1}$ microturbulent velocity, Kurucz (1974) derived a theoretical 
blanketed model photosphere which is in fairly good agreement with the HSRA model, up to the region of the temperature minimum.

Using eclipse data of line intensities, Van Dessel et al. $(1973,1974)$ found a good agreement with the HSRA model, including empirically Holweger's excitation temperature. Data of the 1973 eclipse are also to be published (Houtgast and Namba). Atomic lines were used for detailed analysis by Altrock et al. $(1972,1973,1974)$. The $\mathrm{Mg}$ I 4571 line, well represented by a LTE source function, leads to a slightly modified HSRA model (temperature minimum equal to $4140 \mathrm{~K}$ ). An optimal model atmosphere was derived by a trial and error method from a simultaneous analysis of 3 lines of $\mathrm{Mg} \mathrm{I}$, up to the altitude $1000 \mathrm{~km}$. Taking into account the partial frequency redistribution in the wings of $\mathrm{Ca}$ II resonance lines, semi-empirical models have led to a $4350 \mathrm{~K}$ temperature minimum (Shine et al., 1975; Ayres, 1975; Ayres et al., 1975).

Extensive works have been carried out in order to derive the temperature in the upper photosphere from molecular line spectra. The analysis of the CO lines near $\lambda=5 \mu$ was suggested by Berger et al. (1972) because of the high-temperature sensitivity, the depth of formation and a plausible LTE assumption. Observation by Noyes et al. (1972), Goldman et al. (1973), Müller and Sauval (1975) agree approximately with the HSRA model. Nevertheless, large-temperature fluctuations (with temperatures as low as $3500 \mathrm{~K}$ ) have been detected, which support the idea of large inhomogeneities in this region. $\mathrm{CN}$ and $\mathrm{CH}$ systems have been studied by Mount et al. $(1973,1974 a, 1974 b)$. The corresponding best-fit models are a little cooler than the HSRA in the deep layers, and a little hotter near the temperature minimum. Non-LTE aspects have been examined in the case of $\mathrm{CN}(0,0)$ by the same authors (1975). Other molecular lines investigated by Grevesse et al. (1973) are those of $\mathrm{C}_{2}, \mathrm{CH}, \mathrm{NH}, \mathrm{OH}, \mathrm{Mg}$ II. The results disagree with the HSRA only in a few cases. New laboratory measurements could perhaps explain the discrepancies.

Faculae models have been derived by Caccin et al. (1974), Shine et al. (1974). Effects of geometry on the centre-to-limb contrast are currently under study (Caccin et al.).

\section{Line Profiles}

The understanding of line formation mechanisms was improved by new results of theoretical atomic physics. More accurate calculations of oscillator strengths have been obtained in connection with abundance determinations (see below). Collisional broadenings were investigated with a Smimov-Roueff interaction potential in the case of light neutral perturbers. An application to some infra-red lines shows a better agreement with observations than in the case of pure van der Waals interaction (Deridder et al., 1974; Van Rensbergen et al., 1975). Calculations using results by Lewis et al. have been carried out in the case of the Na D lines (Blackwell et al., 1972; Worral, 1973). Elste et al. (1973) pointed out the need of a good Stark broadening theory in order to interpret the Balmer line wings.

Attempts have been made to explain the line-shifts at the limb. Using a Lennard-Jones potential, Hart (1974) obtained a good agreement with observations.

The empirical determination of the source function has been attempted by new techniques. Hearn and Holt $(1973,1974)$ used simultaneously the single line and the centre-to-limb inversion for $\mathrm{Na} \mathrm{D}_{2}$. In the case of LTE, such a determination leads to the temperature profile of the model, as seen previously (Altrock et al.). The Phillips-Twomey method was shown to be particularly well-suited to the problem of inversion of the limb-darkening equation in the presence of noise (Kunasz et al., 1973). Using many lines mainly of high excitation potential, De Jager et al. (1972) determined source functions, departures from LTE and micro-turbulent velocity models.

Various elements have been studied from the point of view of a non-LTE analysis: Na I(Gehren, 1975), Mg I(Altrock and Canfield, 1974), Al I (Finn and Jefferies, 1974), O I (infra-red triplet, Sedlmayr, 1974). For Fe I, van Dessel (1974) used Holweger's excitation temperature to interpret eclipse data, while Athay and Lites (1972) predicted, with a 15-level reference atom, departure from LTE partly checked by Smith (1974). Eclipse limb spectra of Ba II 4554 are presently being studied by Rutten, and show that complete redistribution 
cannot be assumed, even in the case of moderately strong lines. The $\mathrm{C}_{2}$ singlet states of Phillips bands were shown to be populated in LTE by Sinha and Pande (1974). The influence of blends in transfer calculations has been investigated by Lites (1974). Gebbie and Steinitz (1973) have shown that contrasts in radiatively controlled lines (Balmer lines for example) may arise from lateral differences in the local value of the scattering terms. Correlations between intensity fluctuations in various lines (Evans and Catalano, 1972) imply source function problems (Sivaraman, 1974). Looking into the formation of lines in a multidimensional medium, with lateral radiative transfer, Jones (1973) presented numerical results for the corresponding source-function. We shall discuss later the problems connected with macro- and micro-turbulence.

Contribution functions referring to emergent radiation and line depression have been compared (Sorli and Engvold, 1972; Gurtovenko et al., 1974). For weak lines, the latter is most relevant. We also note that the sensitivity of selected lines with respect to the local effective temperature of the atmosphere has been listed by Caccin et al. (1973) with a view to poleequator and magnetic-field effects.

\section{Polarization}

The polarization of the solar continuum was observed in various wavelengths ranges. Near $\lambda$ 5834, Milkey and Orrall (1974) measured the four Stokes parameters of radiation. The linear polarization obtained is in good agreement with theoretical predictions by Debarbat et al. (1970) and Dumont and Pecker (1971). Leroy (1974) found a polarization rate which decreases strongly from 6000 to $8000 \AA$, and then remains constant up to $9800 \AA$. In the far infra-red range $(100 \mu-2000 \mu)$, measurements have been carried out with balloon-borne experiments and from the ground (Dall'Oglio et al., 1973, 1974) showing that the polarization does not depend on wavelength.

Polarization in line wings was theoretically studied by Dumont et al. (1973). The predictions were qualitatively confirmed by Stenflo's measurements in Ca I 4227. The depolarization mechanism is sensitive to the collisional damping constant, and we notice that a better agreement is obtained with the recent value of Lites (Stenflo, 1974). New line and continuum polarization measurements have been obtained recently (Wiehr, 1975). Theoretical computations for line cores are in preparation (Dumont et al.). A new list of maximum polarization according to $J$ values has been given by Beckers (1974).

\section{E. Unresolved Inhomogeneities}

The unresolved inhomogeneities divide roughly into two kinds: the 'micro' ones (radial size smaller than the width of some 'contribution function' corresponding to the observed quantity) and a part of the 'macro' ones, (radial size greater than this width), the tangential size of which is too small to be resolved by the instrument. These notions depend strongly on the quantity used for the diagnosis (continuum, lines) and on the spatial resolution of the observations (from a few hundreds $\mathrm{km}$ to the whole Sun). Following the literature, we restrict the discussion to the case of velocities.

Theoretical works using stochastic processes and which deal simultaneously with all the possible sizes of inhomogeneities have been carried out. Centre-limb variations of line Doppler width have been interpreted (Auvergne et al., 1973; Gail et al., 1974; Frish H., 1975): the correlation length for the vertical velocity distribution appears to lie between 70 and $300 \mathrm{~km}$. Non-LTE calculations by Wilson and Guidry (1974) have shown that the centre-tolimb increase of the Doppler width cannot be caused by frequency dependence of the source function. They have suggested hot and cold columns to account for the limb observations.

Asymmetries of average line profiles can occur in the case of propagating waves (Kostyk and Orlova, 1972) or ascending and descending bubbles (Schatzman and Magnan, 1975) which account for the observed correlation with the strength of the lines (Kostik and Orlova, 1974). It is pointed out by Magnan and Pecker (1974) that the use of line bisectors to determine the velocity field may be very misleading. 
De Jager (1972) assumed that macro- and micro-motions were observed through filters defined by line shifts and line broadenings. Computing these filters and using observed ratios of macro- and micro-velocities, he has shown that the motions are contained in a small range of wave numbers, corresponding to granular cell diameters. Using a deconvolution method, Rutten et al. (1974) derived a non-gaussian velocity distribution, not incompatible with Kolmogoroff's law. De Jager's calculations (1974) for given turbulence spectra have also been compared to the line profiles. Some others works have been devoted to empirical determinations of the amplitude and variation with height of anisotropic macro- and micro-velocity fields (Lites, 1973; Troyan, 1973; Kondrashova et al., 1974; Altrock et al., 1974).

The problems connected with unresolved motions are very difficult. In our opinion, the solutions could be improved if the various phenomena involved (penetrative turbulent convection, waves) could be included in the calculations with their own properties, for example with realistic phase lags between velocity and temperature.

\section{F. Very Large-Scale Structure, Pole-Equator Effects}

The general theory of relativity is connected to the problem of the solar oblateness. Dicke $(1972,1974 \mathrm{a}, 1974 \mathrm{~b})$ observed the value $\Delta r / r=4.5 \times 10^{-5}$ and a periodicity equal to 25.7 days. These measurements have been the subject of controversy. Some other mechanisms could mimic the oblateness: presence of faculae (Chapman and Ingersoll, 1972, 1973, 1975; Dicke, 1972, 1974), anisotropy of the granulation pattern (Kandel et al., 1973), small differences between the polar and equatorial limb darkening function (Hill et al., 1974). Effects of equatorial temperature excess due to mechanical waves (Ulmschneider-Kalkofen, 1973) have been investigated. Anyway the equator-pole temperature difference is probably too small to mimic the measured oblateness (Canfield, 1973; Noyes et al., 1973; Dicke, 1973; Rutten, 1973; Peraiah, 1973; Falciani et al., 1974).

Differential rotation and large-scale currents are important for problems of the solar interior and the convection zone. New spectroscopic data were available (Howard, 1973; Plaskett, 1973; Hendl, 1974). Sunspot observations have also been analysed (Knoska, 1975). A small negative correlation is found between the rotational velocity and solar activity (Howard, 1973). Livingston and Milkey (1972) have attempted to detect a photospheric height gradient. Discussions about solar rotation can be found in the review paper by Gilman (1974).

The very large-scale solar magnetic field was analysed by using data over long periods. The average EW inclination of field lines has been measured by Howard (1974). A detailed analysis in terms of surface harmonics was given for the years 1959-1972 by Altschuler et al. (1974).

\section{G. Spatially Resolved Inhomogeneities}

The field of spatially resolved structures was greatly extended during the last few years mainly because of the availability of extremely good observations. The 'boundary conditions' for accurate theoretical predictions are now well defined. Some review papers are available from both the observational (Beckers, in preparation) and the theoretical (Stein and Leibacher, 1974) points of view.

A few works have been devoted to supergranulation. Very long lifetimes and temperature decrease in the very deep layers of the cell boundaries have been suggested by Worden (1975).

The granulation was again analysed by using Stratoscope data (Edmonds $e t$ al., in preparation). A high value of the rms of brightness fluctuations was obtained. A special study of low wavenumber power was carried out, showing that the granulation is essentially due to turbulent thermal convection (Edmonds, in preparation). Photographs taken with narrow-band filters in the center of $\mathrm{Fe} I 6569$ and in the continuum indicated that the granules are smeared in the enhanced network (Bray et al., 1974). Speckle interferometry was applied to granulation by Harvey and Schwarzchild (1975), leading to the detection of details smaller than 0.3", and methods of statistical analysis have been reported by Aime and Ricort (1975). Attempts to build inhomogeneous models of the photosphere were made by Turon (1975) using infra-red data, and by Levy (1974) taking into account the horizontal transfer of radiation. Altrock and 
Musman (1975) derived temperature fluctuations from line profiles and found that they are largely controlled by radiation in the low photosphere.

In order to derive velocity fields from line shifts, weighting function for velocities (Mein, 1971 ) or the so-called response functions (Beckers and Milkey, 1975) are very helpful. Problems of noise and finite spectral and spatial resolution have been investigated by Brandt $e t a l$. (1973) and Mehltretter (1973). Influence of weakening and saturation of lines in the presence of the magnetic field have also been pointed out (Frazier, 1974). Horizontal velocities have been derived from Doppler shifts and proper motions by Dravins (1975). Livingston and White (1974) observed horizontal velocities at the limb and found different behaviours according to the involved lines. Let us turn now to the predominantly vertical velocities as observed on the disk and investigated in numerous extensive works.

Purely spatial analysis have been carried out with a view to determine isotropy (Gopasyuk et al., 1972), size of cells (Teske, 1974; Lynch et al., 1975), variation of amplitude with height (Canfield and Mehltretter, 1973), attempt to discriminate granulation and oscillations by scale consideration (Mattig and Nesis, 1974). The stability of wavenumber spectra has been discussed (Deubner, 1974). The oscillations, as observed at one point of the Sun during a long time interval are very similar to a narrow-band random process (Cha and White, 1973). It must be noticed also that temperature fluctuations associated with oscillations in the high photosphere have been measured through infra-red observations in the CO-line (Noyes and Hall, 1972) and in the continuum (Hudson and Lindsey, 1974).

Various techniques have contributed to the two-dimensional study - space and time - of the oscillations: atomic beam for high spectral resolution (Fossat et al., 1974; Snider et al., 1974) and very rapid sequences of photographic spectra for high spatial resolution. The power at long horizontal wavelengths is shown to be very large by the first kind of data. Through the simultaneous use of several lines, the second one leads to the study of the vertical propagation (Sivaraman, 1973a, b; Canfield and Musman, 1973). No correspondence was found between granules and oscillation starts (Mussman, 1974). This points out that granulation and oscillations have different origins. For the granulation, a satisfactory agreement was found by Edmonds (1974) between convection flux in the deep photosphere and theories of the convective overshoot. In the higher layers, the best diagnosis can be derived from the $\mathrm{k}-\omega$ diagram. Horizontal sound waves fail to appear (Stix and Wohl, 1974). On the other hand, the analysis of low wavenumber modes, claimed by Thomas et al. (1972) has led Deubner recently (in praparation) to match with high weight statistical data, the acoustic modes trapped in sub photospheric layers as suggested by Ulrich (1970) and the non-radial pulsations of the entire Sun suggested by Wolff $(1972,1973)$. The overstability of the modes which are trapped in the convection zone has been studied by Ando and Osaki (to be published). The results agree with the observed location in the $k-\omega$ plane.

In addition to excitation problems, propagation problems must be solved in the photosphere. The tunnel effect has been studied by Zhugzhda $(1972,1973)$ and small-scale evanescent oscillations predicted by Moore (1974). The response of a bounded atmosphere has been studied by Berthomieu (1974) and Provost (1975). Radiative damping rates have been investigated by Chen (1974) - theoretical predictions in the gravity mode range - and by Schmieder (in preparation) - observations in the acoustic range. Mass transports, such as the material flow in the supergranules, can also be induced by oscillations in the dissipation case (Tjotta-Tjotta, 1974). In the upper photosphere, shocks are expected (Chiuderi et al, 1975). Stein et al. $(1972,1973)$ have shown that low frequency waves can propagate up to high levels of the atmosphere, giving back to the 300-s oscillations some efficiency for the coronal heating.

Velocities in the presence of magnetic field have been observed (Gopasjuk et al., 1972; Teske, 1974). The various domains of trapped waves have been discussed in the $\mathrm{k}-\omega$ plane (Chen $e t a l$, 1972; Nakagawa et al., 1973; Michalitsanos, 1973).

\section{H. Photospheric Magnetic Field}

Various theoretical works have been devoted to line formation in the presence of a magnetic field. Non-LTE approaches (House and Steinitz, 1973, 1975; Domke and Staude, 1973a, b) and 
new methods based on density matrix techniques or perturbation methods (Landi degl'Innocenti et al, 1972, 1973) have been applied to this problem. The case of inhomogeneous magnetic field was also studied (Rachkowsky, 1972). A list of sensitive lines has been published (Harvey, 1973). Temperature effects and saturation effects can account for a slight part of the discrepancies between measurements from different lines (Caccin et al., 1974; Gopasyuk et al., 1973). 'The response of different kinds of magnetographs has been considered by Rees. Depths of line formation were computed for accurate interpretation of the data (Staude, 1972; Wittman, 1974).

The correlation between magnetic structures and the intensity fluctuations in chromospheric lines $\left(D_{3}, H \alpha\right)$ has again been stressed (Chapman, 1972; Gopasyuk-Tsap, 1972; McIntosh, 1972). Some progress has been made on the very important problem of the small scale structure of the magnetic field. New observations with the Kitt Peak multi-channel magnetograph have shown that the field is channelled through very narrow structures (1500 G within 100 or $300 \mathrm{~km}$ ) even in quiet areas (Frazier and Stenflo, 1972; Stenflo, 1973; Chapman, 1974). A comparison with the 'crinkles' discovered by Dunn and Zirkar (1973) in the 'filigree' shows no coincidence with the magnetic footpoints (Simon and Zirker, 1974). Various mechanisms such as turbulent pumping and the Bernoulli effect, have been suggested by Parker $(1974 a, b)$ in order to explain the field concentration in the magnetic filaments.

The time variation of the magnetic field has been observed in surface details by Lynch (1974). Some very long lifetimes (4-7 days) in the supergranular pattern have been found by Livingston and Orrall (1974). On the other hand, the magnetic field of the Sun as a star oscillates with the 300-s period (Joshpa et al., 1973).

Theoretical studies have been carried out in order to give a practical representation of force-free fields and to derive the energy spectrum of the magnetic field from a passive response to turbulent fluid motions (Nakagawa, 1973; Nakagawa and Priest, 1973). The spectrum of large-scale fields and motions of foot-points were also investigated (Nakagawa and Levine, 1974; Low and Nakagawa, 1975).

\section{Solar Radiation and Spectrum}

The problem of absolute intensities and solar constant has been discussed by Neckel and Labs (1973) and Thekaekara (1974). The measurements of absolute intensities in the UV range $(1200-2000 \AA)$ in connection with homogeneous temperature models have also been examined. A photometric atlas by Delbouille, Roland, Neven will cover the 4000-6850 \& domain. The Kitt Peak table of photographic wavelengths has been published (Pierce et al., 1973). For blanketing problems, the fraction of absorption lines has been computed by Wohl (1975) with respect to the wavelength from 3000 to $20000 \AA$. A UV atlas $(2226-2992)$ by Tousey et al. is in print; 700 lines have been listed by Moore in the UV disk spectrum, and a ledger of the limb spectrum has been completed by Tousey. In the IR range $(1-3 \mu)$, data are available from the Arizona NASA atlas (Benner $e t$ al., 1972) and from spectra obtained by Zander, Delbouille and Roland.

Several molecular lines have been identified in the solar spectrum in the case of $\mathrm{C}_{2}$ (Lambert et al., 1974) and $\mathrm{C}_{2}, \mathrm{CH}, \mathrm{CO}, \mathrm{CN}, \mathrm{NH}, \mathrm{OH}, \mathrm{MgH}$ (Sauval et al., 1973, 1975). Telluric blending problems could be avoided by observing the solar spectrum reflected and shifted by a planet (Day, 1974).

\section{J. Photospheric Abundances}

Many problems arise in the accurate determination of abundances. Neglecting NLTE effects can lead to errors by a factor 3 (Dumont et al., in press). Damping constants and microturbulence are also very important (Blackwell et al., 1972).

New oscillator strengths have been calculated for O, C (Nicolaides, 1973), C, Ti, Sc (Biemont, 1973, 1974a, b) and measured for Fe (Assousa et al., 1972; Smith et al., 1973), Cr (Cocke et al., 1973), Mn (Pinnington et al., 1974), Ni (Lennard et al., 1975) and rare earth ions (Ander- 
son et al., 1974). Lists of abundances and isotopes ratios have been given by Engvold et al (1974). The following list gives the references of recent abundance determinations:

He - Hirshberg (1973); Li - Brault et al., 1975; Muller et al., 1975; Be - Aller, 1973; Chmielewski et al., 1975; Ross and Aller, 1974; B - Wohl, 1974; Hall-Engvold (1975); O - Delone $e t$ al., (1973); Si - Holweger (1973); K - De la Reza (in print); Ca - Holweger (1972); Ti - Foy (1975); Mn - Margrave (1972); Fe - Lites (1973); Huber and Tubbs (1973); Ross (1973a, b); Ga - Mutschlecner (1972); Ge - Ross and Aller (1974); Y - Allen and Cowley (1974); $\mathrm{Nb}$ - Hauge and Youssef (1975); Ag - Ross and Aller (1972); Ba-Holweger and Muller (1974); Tm - Ross and Aller (1974); Pb, Th - Hauge and Sorli (1973); Bi - Hauge (1974).

Investigations for isotopic composition have been carried out in the case of $\mathrm{Rb}, \mathrm{Sr}, \mathrm{Eu}$ (Hauge $1972 \mathrm{a}, \mathrm{b}, \mathrm{c}$ ) and Sm (Ekeland and Hauge, 1975). Some attempts have also been made to detect ${ }^{13} \mathrm{C},{ }^{17} \mathrm{O},{ }^{18} \mathrm{O},{ }^{3} \mathrm{He}$ (Hall, 1973, 1975) and also deuterium (Beckers, 1975).

\section{THE CHROMOSPHERE \\ (M. K. V. Bappu)}

\section{A. Spicules}

Lynch et al. (1973) have measured spicule properties on much improved filtergrams obtained with the Sacramento Peak vacuum telescope. The average spicule diameter is $950 \mathrm{~km}$ well above the instrumental resolution of $0.6^{\prime \prime}$. The variations of spicule diameter with height, wavelength and time are uncertain and do not confirm earlier reports of rapid spicule expansion. Direct counts referring to off-band filtergrams (H $\alpha \pm 0.75 \AA ; \pm 1.0 \AA$ ) and to an approximate height of $4000 \mathrm{~km}$ give spicule numbers that are three times larger than those reported before. A real decrease in spicule numbers for low heights explains satisfactorily the appearance of the dark band beyond the photospheric limb described by Loughhead (1969) and confirmed by Nikolskii and others. Mouradian (1974) finds from a study of filtergrams that the most probable value of inclination of spicules in relation to the normal to the solar surface is $25^{\circ}$ and that nearly $62 \%$ of all spicules have inclinations within $\pm 10^{\circ}$ of this value. Escant and Mouradian have in progress a study of spicule spectra on the solar $\operatorname{limb}$ in $K$ and $D_{3}$.

Moe et al. (1975) have compared the spicule-like features well visible in He II 304 A Skylab spectroheliograms with spicules seen in $\mathrm{H} \alpha$ filtergrams obtained simultaneously. No correspondence between the two is seen. However, this does not rule out the possibility that spicules, like prominences, may have differences in appearance when seen in $\mathrm{He}$ II and in $\mathrm{H} \alpha$.

Banos (1973) has studied some properties of spicules at the limb through a K-filter. He finds these features in $K$ to be inadequately resolved as compared to $H \alpha$. The ratio of spicule intensity to background intensity increases with height similar to the $\mathrm{H} \alpha$ behaviour, though less striking. The widths are larger than in $\mathrm{H} \alpha$, though this needs to be re-examined under better conditions of observation. The lifetimes are of the order of 270 secs; these are the same as the lifetime of the fine bright mottles as determined by Bappu and Sivaraman (1971) and from this aspect alone, both may be the same in identity. The velocity of ascent is similar to the average value derived in $\mathrm{H} \alpha$.

Alissandrakis (1973) has analyzed simultaneous spectra of spicules in $H \alpha, H \beta$ and $K$, obtained with a resolution of $1.5^{\prime \prime}$. A very good correlation in the structure of all three lines is seen. The rotation velocities around the spicule axis must be small. Optical depth effects contribute to the $\mathrm{H} \alpha$ line widths. The broadening of the $\mathrm{K}$ line is mostly non-thermal.

Zirin (1974a) has studied $K$ line disc filtergrams and finds that while they produce $K_{3}$ absorption, they occur in emission more often than in $\mathrm{H} \alpha$, especially near the limb. Bright spicules and dark ones are seen over all elements of the $K$ network similar to the $H \alpha$ case. In a study of spicules and fibrils in $\mathrm{H} \alpha$ and $\mathrm{K}, \mathrm{Zirin}$ (1974b) finds near the limb, bright $\mathrm{H} \alpha$ features at the bottom of dark spicules. In $\mathrm{K}$ both bottom and top of the features are bright. These are also bright vertical features in $H \alpha$ that are uniformly bright in $K$ too. 
Bohlin et al. (1975) report a major new characteristic of the Sun's polar cap, which they term polar macro-spicules. In He II $304 \AA$ they appear as long pointed jets ranging in length from $5^{\prime \prime}$ to $60^{\prime \prime}$. The macro-spicules occur only over the polar cap as defined by the polar coronal hole seen in UV spectra. No counterparts in the visible wavelength are seen and in nearly every characteristic they are substantially different from the spicule seen in $\mathrm{H} \alpha$.

\section{B. Supergranulation}

Kubicela (1973) has evaluated spatially the line of sight velocities within a supergranular cell. A tangential component to the horizontal supergranular motion is invoked that can be explained both in direction and magnitude by a Coriolis velocity. Local enhancements of velocity fields, especially of approach, have been observed to give rise to a rearrangement of the new supergranular boundary. The enhanced calcium network that outlines the supergranular boundary follows the velocity changes in time. Kubicela and Karabin have in progress a programme for measurement of these large-scale photospheric velocities. Detailed studies of supergranular dynamics coupled with simultaneous magnetic field and calcium intensity values are needed to understand the evolutionary aspects of the supergranule.

Giovanelli (1974) has found that, over supergranule centres, the $\mathrm{H} \alpha$ chromosphere has a fine structure at line centre of similar scale to the photospheric granulation but having no geometrical correspondence with it. The chromospheric granulation can be seen wherever the fibrils are absent, or where there are gaps between the fibrils, so that it lies below them. There is also a faint diffuse structure visible in the $\mathrm{H} \alpha$ line core and outlining the network; this may well be the interspicular absorbing material postulated by Michard to account for the central reversals seen in spicule $H \alpha$ profiles.

A first look at the supergranular network from the Skylab scanning UV spectrometer in many wavelengths, that originate from different heights in the solar atmosphere, shows (Reeves et al., 1974) the preservation of spatial identity with changes in contrast, up to excitation temperatures of $3 \times 10^{5} \mathrm{~K}$. It diffuses rapidly and becomes unrecognizable at temperatures of $1.5 \times 10^{6} \mathrm{~K}$, suggestive of a substantial spread in the magnetic-field configuration at these heights. An interesting feature is that individual bright points are seen unchanged in size through this entire range in height and temperature, indicating a localized channelling of magnetic fields through coronal temperatures. Detailed analyses of Skylab data, pertaining to the chromospheric network, its coronal extension and temporal changes in its structure, are in progress at Harvard.

The association of the supergranule boundary with the formation of an active region finds further support in a recent study by Born (1974). A rearrangement of the network pattern is seen with a new cell surrounding the active region.

\section{The Continuum}

Vernazza, Avrett and Loeser (1975) have reviewed all available observations of the UV solar continuum as part of the development of a model of the photosphere and low chromosphere. Listed in measures of intensities and fluxes in the region 2000-3000 $\AA$ are the recent values of Kohl et al., (1975) and of Heath (1973). Since heavy line blanketing occurs in this region one needs to have high-resolution observations to evaluate the continuum level. Tousey et al. (1974) have an extensive compilation for the region $2226 \AA$ to $2992 \AA$. Less extensive lists in the $2800 \AA$ region are those of Greve, McKeith (1973) Greve and McKeith (1974). Recent stigmatic spectra obtained by Samain et al. (1975) show a less pronounced decrease at the Al I photoionization edge than did earlier observations. Vernazza et al. find fair agreement between their model and the observations, provided line blanketing is adequately accounted for. With the use of improved Al I photoionization cross sections measured by Kohl and Parkinson (1973) they find good agreement between the observed and calculated continuum intensities near $2077 \AA$.

Bonnet (1975) has reviewed recent progress in understanding the regions of origin for the emission between $1250 \AA$ to $2000 \AA$. Available flux measures in this region are by Nishi (1973), 
Samain et al. (1975), Rottman (1974), Heroux et al. (1975) and by Jordan and Ridgeley (1974). A compilation of low-resolution observations has been completed at the U.S. Naval Research Laboratory and high-dispersion spectra are currently being reduced. McAllister and Smith (1975) also list lines observed at high resolution in the vicinity of the Al I autoionizing lines between $1889 \AA$ and $1969 \AA$. Line blanketing has to be evaluated carefully to have a good match between theory and observation. This presents the problem of the 'missing opacity'. Vernazza et al. (1975) have taken account of line blanketing through the use of the extensive calculations of Kurucz (1974) and Kurucz et al. (1974). Line-blanketed models have also been discussed by Petreymann (1974). However, it does appear that some opacity is still underestimated. Landi Degl'Innocenti and Noci (1973) consider negative ions other than $\mathrm{H}^{-}$, two photon processes, forbidden dissociation of $\mathrm{H}_{2}$ molecules, photodissociation of molecules and absorption by quasi-molecules, but find them insufficient contributors. However, Drake (1974) has suggested that electron-hydrogen photo-attachment giving rise to the $2 p^{2}{ }^{3} P$ state of $\mathrm{H}^{-}$ may be important, and Krishnaswamy and Stecher (1974) have proposed $\mathrm{H}_{2}^{+}$in non-LTE as a possible source.

In the region $1500 \AA$ to $1700 \AA$ where the apparent brightness temperature passes through a minimum value, the recent observations now give agreement around $T_{b}=4400 \mathrm{~K}$. Vernazza et $a l$. have shown the importance of non-LTE calculation for the principal opacity source, the Si I continuum. They suggest that non-LTE effects cause the observed brightness temperature to be higher than the electron temperature value of $4200 \mathrm{~K}$ derived from infra-red observations. The recent work of Brown et al. (1974) in the laboratory and Moore et al. (1975) should greatly help extended studies of $\mathrm{Si} I$ level populations. Further progress in understanding the opacity sources is needed before differences between observed and theoretical centre-to-limb intensity ratios can be ascribed to departures from the spherically symmetric homogeneous model. However, inhomogeneities will have to be incorporated in future realistic models. Jordan and Ridgeley (1974) find that limb-to-disk observations made with the limb slit 4 " above the limb show that continuous emission from material with $T_{b} \leqslant 5000 \mathrm{~K}$ extends to heights of between $2000 \mathrm{~km}$ to $5000 \mathrm{~km}$ beyond the limb, values far in excess of $700 \mathrm{~km}$ predicted by HSRA for this temperature. This value is also above the average height of the chromosphere-corona transition region as derived from optically thin emission lines.

Kurokawa et al. (1974) have analyzed flash spectra of the 1970 eclipse. Distributions of the surface brightness of the continuum at six wavelengths in the visible region show a shallow dip and a small hump that cannot be reproduced by any of the existing model atmospheres. Linsky (1973) has recalibrated solar microwave flux measurements $(1 \mathrm{~mm}-3 \mathrm{~cm})$ using the Moon as an absolute radiometric standard. This will be useful for comparison with predictions of solar chromospheric models. Foukal (1974) considers a three-component concept to describe present observations of the chromosphere and transition region in EUV, optical and mm wave-lengths. The three components are the interiors of supergranular cells, the hot plagettes overlying faculae, and the cooler transient mottles which surround them in the network boundaries. Van Dessel (1974) points out the improvement made by considering non-LTE effects in $\mathrm{Fe} I$ line formation with variation having a height dependence.

\section{Line Profiles and Spatial Inhomogeneities}

The Al I autoionization lines at $1932 \AA$ and $1936 \AA$ have been observed by McAllister (1974) in a centre-to-limb study with $3^{\prime}$ spatial and $0.01 \AA$ spectral resolution. Theoretical calculations by Finn and Jefferies (1974) of these line profiles show the non-LTE formulation to have better fit with either the Bilderberg or HSRA model, than an LTE calculation. However, a greater continuum opacity is needed than what is normally accounted for, in order to fit the limb-to-disk variation of the profiles.

The $\mathrm{Mg}$ II lines at $2796 \AA$ and $2803 \AA$ have been extensively studied in recent years. Lemaire and Skumanich (1973) report on profiles observed with 7 " resolution and moderate $(0.25 \AA)$ spectral resolution, and show examples of profiles in the supergranulation network, in cellcentres and in plage regions. Current models do not give a good fit to the profiles and it is difficult to deduce unambiguously which specific parameters in the models need to be 
modified. Gouttebroze and Lemaire (1974) have analyzed these profiles and find that they are incompatible with those produced in a spherically symmetric atmosphere in which the source function is independent of frequency. The observations would suggest the presence of nonthermal motions of between $9-20 \mathrm{~km} \mathrm{~s}^{-1}$, preferentially, in the vertical direction. Milkey and Mihalas (1974) have made a detailed study of the Mg II profile assuming partial rather than full redistribution in the line profile and improve the fit between observation and theory. They also suggest that a better theoretical value for the van der Waals broadening in $\mathbf{M g}$ II is needed before the line profiles can be used to refine existing solar models. Centre to limb observations including the $\mathrm{Al} I$ and $\mathrm{Mg}$ II lines have also been reported by Kohl et al. (1975).

Observations of profiles of lines of C I, S I, C II, A III, Si II and Fe II have been made by Boland et al. (1973), (1975) with a spatial resolution of $\simeq 40^{\prime \prime}$ and high spectral resolution $(0.03 \AA)$. Lines that are estimated to be optically thin show approximately Gaussian profiles with widths that correspond to non-thermal velocities of between $4 \mathrm{~km} \mathrm{~s}^{-1}$ and $7 \mathrm{~km} \mathrm{~s}^{-1}$. Lines which on the basis of current models would be expected to have high optical depths show pronounced self-reversals. The interpretation of these lines is complex because of the large range in height and temperature of the region formation. Jefferies and McAllister (1973) have also reported observations of profiles of $\mathrm{Si}$ II lines and Finn and McAllister are currently engaged on a non-LTE analysis of these and other Si II line profiles. Bruner et al. (1973) have also obtained line profiles of $L y-\alpha$ and of the $O I$ lines at $1304 \AA$ and $1306 \AA$ with $0.01 \AA$ and $20^{\prime \prime}$ resolution. These show the correlation of decreased central reversals in these lines with bright regions of the calcium network. Milkey and Mihalas (1973) have also calculated the Ly- $\alpha$ profile allowing for partial redistribution and find again a better fit to the observations than by using full redistribution.

Livingston and White (1974) describe a spectrum obtained in very good seeing in the $5870 \AA$ region that probes the high photosphere through the low chromosphere. The stronger emission lines show up velocity and brightness structures with sizes ranging from 500 to $1500 \mathrm{~km}$. The emission knots in $\mathrm{Fe} \mathrm{I}$ and $\mathrm{Ba}$ II lines coincide with continuum bright streaks. No correlation is evident however, between structure in $\mathrm{He} \mathrm{I} D_{3}$, emission in $\mathrm{NaI} \mathrm{D}_{2}$ and emission in the $\mathrm{Fe} I$ and $\mathrm{Ba}$ II lines as a group. Differences in line opacity are suggested as the cause of the low correlation between the fine structure in the various lines.

From studies of the infra-red Ca II triplet, Mein (1971) finds a three-column model to describe the steady field of temperature, micro-turbulence and radial velocity fluctuations in the photosphere-chromosphere transition zone.

Gebbie and Steinitz (1974) have investigated patterns in H $\alpha$ spectroheliograms, and conclude that the $\mathrm{H} \alpha$ line source function in the quiet chromosphere is indirectly controlled by the photospheric radiation fields in the Balmer and Paschen continua. Variations in the shape of the absorption profile are extremely effective compared to changes in the source and sink terms. Mouradian has in progress the simulation of the chromosphere by the Monte-Carlo method. In computing the emergent intensity of chromospheric origin at the limb, energy transfer in an inhomogeneous atmosphere is taken into account with different physical parameters having different distribution functions.

From a study of contrast of bright and dark mottles in $\mathrm{H} \alpha$ as a function of wavelength, Bray (1973) finds it to be in good agreement with Becker's 'cloud' model. The values of source function and optical thickness derived seem consistent with Becker's spicule model. However the spicule model finds little agreement with observations when similar measures of contrast are carried out at the limb (Loughhead, 1973). The bright mottles seen beyond the limb reveal no evidence of a systematic upward or downward motion persisting throughout the mottle lifetime (Loughhead, 1974) though a short period vertical oscillation of low velocity is not excluded.

Mein and Mein (1975) have derived intensity curves at different wavelengths in limb spectra of $\mathrm{H} \alpha, \mathrm{H} \beta$ and $\mathrm{H} \gamma$. Between 2000 and $6000 \mathrm{~km}$ above the limb the average thermal, together with turbulent, velocity of the atoms increases from $20 \mathrm{~km} \mathrm{~s}^{-1}$ to $30 \mathrm{~km} \mathrm{~s}^{-1}$. The Meins aim to derive an inhomogeneous model chromosphere. Sivaraman and Venkitachalam (1975) have completed a study of intensity fluctuations as seen in the first four members of the Balmer series. The dependence of the rms brightness fluctuations on heliocentric angle and $\Delta \lambda$ positions within the line profile, as well as the contributions, by structures of different sizes, to these 
fluctuations are estimated.

There has been interest in recent years in the study of the Ca II K line on the solar disc. Punetha (1974a) has studied rms as well as power and coherence spectra of intensity variations. Grossmann-Doerth et al. (1974) present results that improve available information on statistical steady-state conditions as inferred from $\mathrm{K}$ spectra. In the supergranule interior, the profiles with violet single peak, the double peak and no peak at all dominate; in the cell boundary the double peak is most common. In both locations the red single peak is relatively rare. The comparison of the single peak line profiles with model computation shows the presence of non-uniform large-scale velocity fields in the chromosphere. In discussing the formation of Ca II K, Vardavas and Cram (1974) point out the need to consider the frequency dependence of line source function in quantative models. Ayres and Linsky (1975a) have analyzed the Mg II resonance line profiles as well as those of $\mathrm{Ca}$ II with the aid of a partial frequency redistribution formulation. They give strong evidence for a temperature minimum near $4350 \mathrm{~K}$ and a top chromospheric pressure of $0.15 \mathrm{dyne}^{-2}$.

Ulmschneider (1974) has on the basis of HSRA evaluated the radiation loss of the solar chromosphere. He considers the short period acoustic wave theory to be a sufficient mechanism to balance the chromospheric radiation loss.

Harvey, Hall and Giovanelli have studied the $10830 \AA$ helium line, concluding that the anomalous ratio of the strengths of the two components is due, in the weakest regions, to faint uncorrected blends; elsewhere, the relation between the intensity and the anomalous ratio of the two components can be explained only if the absorbing elements are resolved incompletely. In conformity with many eclipse observations, they find that the widths of $10830 \AA$, Py and Ca II $8542 \AA$ are compatible only if they are effectively non-thermal out to at least $5000 \mathrm{~km}$, the rms velocities being some $20 \mathrm{~km} \mathrm{~s}^{-1}$. Milkey has found that He II $304 \AA$ photons may provide a reasonably effective means of pumping the He I ionization in the regions where the $10830 \AA$ line and other observable transitions are formed. Streete and Tandberg-Hanssen (1974) have obtained the helium triplet/singlet line intensity ratio $I_{10830} / I_{20581}$ at the eclipse of 1973, June 30. A lower limit of 60 was found indicating a relative overpopulation of the $2^{3} P$ level.

Harvey in collaboration with several ATM teams has examined ground-based observations of helium lines with simultaneous X-ray observations from American Science and Engineering and EUV slitless spectrograms from the Naval Research Laboratory. These support the idea advanced by Goldberg in 1939 that these helium lines are significantly controlled by radiation arising at much higher temperatures than the regions where helium is observed. As a result, it is possible to detect coronal holes and bright points with helium observations. These phenomena appear to be basically magnetic in origin. The exact association of magnetic fields with coronal holes remains elusive. Studies by AS \& $E$ of X-ray bright points and by Harvey, Harvey and Martin of short lived bipolar magnetic regions definitely confirm the X-ray bright points to be associated with small magnetic active regions. Harvey's study of $10830 \AA$ spectroheliograms shows that polar coronal holes remain strong but equatorial coronal holes have nearly vanished as solar activity continues its decline toward sunspot minimum; this is suggestive that coronal holes, except at the poles, are one of the last vestiges of solar activity.

\section{E. Temporal Variations}

Temporal variations in the $\mathrm{K}$ line have been analysed by Punetha (1974b) from a short time series. Nicole Mein is presently analysing a very good time sequence obtained simultaneously of $\mathrm{H} \alpha$ and the $\mathrm{Ca}$ II lines $(\mathrm{H}, \mathrm{K}, 8498,8542)$ for the study of propagation of waves with a period much less than one minute. Liu (1974) finds intensity perturbations in the $K$ line that propagate from the far wing towards the line core. The perturbation is a local heating in the chromosphere due to an upward propagating disturbance generated in the lower layers. Liu and Skumanich (1974) have synthesized K-line profiles for the different evolutionary stages with physical parameters specified as functions of height. A temperature disturbance matches the observations well, though the mode of heating needs to be established. The disturbance may very well be magnetosonic. The study of evolutionary behaviour (Cram, 1975) of the emission 
in $\mathrm{K}$ and $8542 \AA$ shows the localized enhancement to be associated with an outwardly propagating wave. Beckers and Artzner (1974) have studied the properties of the dark structures seen in the $K_{1}$ line wing using those that move outwards in the solar atmosphere with appreciable velocity. These dark features seem related to the bright ones found by Liu, and both may be related to the shocks that heat the chromosphere and corona. Sivaraman and Venkitachalam have a similar study in progress of the bright points as seen in the $\mathrm{H}$ line of $\mathrm{Ca}$ II. The simultaneous study of the impulse as seen in a neighbouring Fe I line enables the travel of the impulse to be seen with height in the atmosphere. The average lifetime of the bright points is $100 \mathrm{~s}$ and at the conclusion of the brightness enhancement a dark streak is seen which also disappears in about the same time.

The type of waves to be observed in the quiet $\mathrm{H} \alpha$ chromosphere has been studied by Giovanelli (1975a) using methods which show their line-of-sight velocities (i) the chromospheric granulation exhibits vertical velocities of a quasi-oscillatory character but without any horizontal propagation, the characteristic periods being upwards of $2.5 \mathrm{~min}$. Adjacent points appear to have quite independent periods. There is a close relation between intensity at line centre and velocity maximum $v$ occurring about $T / 4$ after maximum downward $v$. The correlation is characteristic of a standing wave and suggests that little energy is transferred into the corona. (ii) Non-sinusoidal waves propagate predominantly loutwards along mottles and fibrils from as close as $2000 \mathrm{~km}$ to the network axis at propagation speed of around $70 \mathrm{~km} \mathrm{~s}^{-1}$. The line-of-sight component of the displacement velocity is $\simeq 5 \mathrm{~km} \mathrm{~s}^{-1}$. The velocities are accompanied by propagating intensity fluctuations. Similar waves have been seen propagating mainly outwards along superpenumbral fibrils. The system appears to be basically of the Alfvén type and carries enough energy up into but not down from the higher regions, where it is presumably dissipated, to account for coronal heating.

\section{F. The Sun as a Star}

In a programme to measure long-term variability of the solar $\mathrm{Ca}$ II $\mathrm{K}$ line, White and Livingston have found that the structure of the $\mathrm{K}$ line is most symmetrical at solar maximum. To relate this result to new OSO-8 data, White is also searching for correlation between the Ca II K and Mg II K (2796 A) lines as a function of activity on the Sun's surface. It may then be possible to observe 'solar cycles' on other stars by means of satellite monitoring of stellar $\mathrm{Mg}$ II $\mathrm{K}$ lines. Bappu and Sivaraman have monitored photographically the $\mathrm{K}$ line in integrated sunlight for nearly half a cycle. The $K_{2} V$ emission continues to be greater that $K_{2 R}$ during this period. The widths measured by a micrometer, as in the stellar case, are larger than in the case of the bright fine mottles over the centre of the disk (Bappu and Sivaraman, 1971). This is principally due to solar rotation and demonstrates the need to evaluate the rotation characteristic in the stars along with the K-line widths, especially in the calibration with absolute magnitude.

Macris (1974) finds the intensity of $\mathrm{K}_{232}$ chromospheric flocculi to background chromosphere at the centre of solar disk, to be 1.482 . This value is of interest to those monitoring total $K_{232}$ intensity as a function of solar cycle. Ayres and Linsky (1975b) have studied the formation of $\mathrm{H} \epsilon$ in the Sun and in $\alpha$ Bootis. In the Sun, the line is in absorption and the source function is dominated by the Balmer continuum (formed in the photosphere) through photoionization. In $\alpha$ Bootis the Balmer continuum radiation field is formed partly in the chromosphere, producing $\mathrm{H} \epsilon$ emission in the wing of the Ca II $\mathrm{H}$ line.

\section{THE TRANSITION REGION AND INNER CORONA (C. Jordan)}

\section{A. The Structure and Heating of the Quiet Atmosphere}

The starting point for many models of the temperature and density structure of the transition region and inner corona, when made from EUV observations, is the distribution with temperature of the emission measure $\int_{R} N_{\mathrm{e}}^{2} \mathrm{~d} h$, which can be derived from each spectral line. Further 
observations of the spatially integrated emission measure distribution in the temperature range $10^{5}-2 \times 10^{6} \mathrm{~K}$ have been made by Malinovsky and Heroux (1973). The earlier work by Withbroe (1970) which describes models made from the emission measure distribution in terms of three parameters, $F_{\mathrm{c}}$, the conductive flux from the corona back through the transition region, $T_{\mathrm{c}}$, the coronal temperature, and, $P_{\mathrm{O}}$, the transition region pressure, has been applied by Withbroe and Gurman (1973) to some 250 spectra obtained from OSO 6, to investigate further inter-relationships between the physical parameters. They find that at pressures $P_{0}$ $\leq 3.0 \times 10^{15} \mathrm{~cm}^{-3} \mathrm{~K}$, the conductive flux, $F_{\mathrm{O}}$, varies approximately as $P_{\mathrm{O}}^{1.8}$, but is constant at higher pressures. Transition region models have also been discussed by Dubov $(1974,1974)$ and by Dubov and Kartoshova $(1973,1974)$. Jordan (1975a) has used the observed emission measure distribution, the equations of hydrostatic equilibrium and conductive flux to derive simple analytical inter-relations between $F_{\mathrm{c}}, T_{\mathrm{c}}$, and $P_{\mathrm{o}}$. Flower and Pineau de Forets (1974) have assumed that line-width observations (Boland et al., 1973) represent wave motions with an associated pressure term which dominates the pressure balance through the transition region, and therefore, have re-analysed the emission measure between $10^{5} \mathrm{~K}$ and $10^{6} \mathrm{~K}$ in terms of a constant electron density rather than a constant electron pressure. Billings and Alvarez (1975) have made models of quiet and active regions from the emission measure distribution and have determined an analytical expression for the variation of $T_{\mathbf{e}}$ with height, which gives interrelations between $P_{O}$ and coronal temperatures. Chiuderi and Riana (1974) have used the hydrodynamic equations and a semi-empirical relation deduced from radio observations to construct a model which agrees quite well with radio and EUV observations and with the solar wind particle flux; the dynamic effects are found to be small.

Whatever the mechanism of heating, Giovanelli (1975b) has pointed out that all heat generated in closed magnetic tubes passing through the corona must be radiated away, though conduction may transfer much heat to the transition region prior to emission. The rate of conduction depends on the tube cross-section which may be assessed by balancing the total pressure within and external to the tube while the coronal density adjusts itself so as to radiate away all heat generated within the tube. For a static model, the observed rate of heating, the computed densities and pressures, the ratio of coronal to transition region emission, and the distribution of radiation in the EUV line spectrum agree closely with those observed.

An alternative approach to making models is to assume a distribution for the mechanical energy deposition and then to construct a model using energy balance and other assumptions, such as hydrostatic equilibrium. Such models can then be used to calculate the emission measure, which can be compared with observations. (See also Section on Corona for further theoretical work). Schmeleva and Syrovatskii (1973) have shown that in the situation where mechanical energy is deposited high in the corona, the structure of the atmosphere can be characterised by two universal functions, one giving the relation between the energy flux deposited and the boundary temperature and the other the temperature variation with height. Landini and Monsignori Fossi (1975) have made models for sets of flux tubes by assuming constant pressure, an exponential fall off in the mechanical energy $\left(F_{m}\right)$ deposition, and $F_{\mathrm{m}} \propto P_{\mathrm{o}}$, and can account for the general observed properties of quiet and active regions. McWhirter et al. (1975) have assumed that the mechanical energy dissipated originates from 300-s period sound waves damped by thermal conduction, and have then set up the energy balance. Gabriel $(1974,1975)$ has constructed a two-dimensional model of the transition region based on the calculated divergence of magnetic-field lines originating from the supergranulation boundaries and energy balance along particular field lines. A model for the supergranulation boundary region has also been derived by combining the observed integrated emission measure with the calculated area occupied by the boundary. The transition region in the boundary model occurs at a greater height and is thicker than in the average models, but the electron pressure is very similar in each case. Livshits and Badaliyan (1974) have considered heat spreading in a gas column with boundary temperatures $10^{6}$ and $10^{4} \mathrm{~K}$ and with time independent hydrostatically changing density. They obtain an estimate of mass of gas flowing out of the chromosphere to the corona.

The atmospheric structure may be studied from the relative intensities of lines as a function of position across the disk and above the limb. Earlier observations of limb-to-disk intensity 
ratios made from rockets have been extended to include measurements of the average height of the optically thin transition region lines (Burton et al., 1973). A height of $1700 \pm 700 \mathrm{~km}$ was observed from in-flight recording of the spectrograph slit position, and $2100 \pm 850 \mathrm{~km}$ from measurements of line images in a stigmatic part of the spectrum. These observations cannot give the variation of the height of the transition region, but optically thick lines show the presence of material at $T_{\mathrm{e}} \sim 1-2 \times 10^{4} \mathrm{~K}$ out to $\sim 10000 \mathrm{~km}$, consistent with the presence of spicules. Brueckner and Nicolas (1973) have studied the behaviour of the transition region lines observed from a rocket during the March 1970 eclipse and have deduced that the transition region must extend around inhomogeneities which extend from 4000 to $11500 \mathrm{~km}$ above the average transition region. The decrease in the emission as a function of height was consistent with the inhomogeneities being spicules. Simon et al. (1974) have analysed spectroheliograms obtained from a Naval Research Laboratory rocket flight and which have a spatial resolution of $3-5^{\prime \prime}$. By comparing the positions of many features in the spectroheliograms in images of five different lines formed at different temperatures they have derived the heights of the lines relative to that of He I $584 \AA$. Their results support a sharp chromosphere-corona interface. Loulergue and Nussbaumer (1974) question the validity of the notion of a very thin chromosphere-corona transition zone, based on the investigation of intensities of C III $977 \AA$, $1176 \AA$ and $C$ II $1334 \AA$. The gas pressure of at least part of the transition zone is insufficient to support the coronal pressure. Mariska and Withbroe (1975) have studied the limb brightening of the EUV lines as observed with the Harvard instrument on the Apollo Telescope Mount (ATM) on Skylab. They find a lower quiet sun temperature than often used, $T_{\mathrm{c}} \sim 1.1 \times 10^{6} \mathrm{~K}$ rather than $T_{\mathrm{c}} \sim 1.5 \times 10^{6} \mathrm{~K}$. They have made an inhomogeneous model for the transition region, finding that the temperature gradient is a factor of three lower at supergranulation boundaries than within the cells. They also find it necessary to include spicules to explain the presence of relatively cool material at heights of $\sim 20000 \mathrm{~km}$ above the limb. Kastner et al. (1974) have observed the behaviour of the lines of Fe $1 \mathrm{X}-\mathrm{XV}$ at heights from $1.05 R_{\odot}$ to $1.3 R_{\Theta}$, using the Goddard spectrometer on OSO 7, and have deduced temperatures from lines of adjacent stages of ionization. The lower temperature near the poles can be detected. Foukal (1974) has proposed a three component model of the chromosphere and transition region composed of supergranulation cell interiors, cool transient mottles (spicules) and hot regions (plagettes) within the supergranulation boundaries. Kanno and Tanaka (1975) have analysed the centre-tolimb variation of the quiet sun at centimeter and decimeter wavelengths and find that the limb-brightening predicted by the models of Brueckner and Nicolas and Foukal is too great. They propose a model where thin platelet transition regions exist on top of the chromosphere, scattered in the network boundaries.

The spatial distribution of the EUV emission lines, which will increasingly be used as input into models, has been observed with improved resolution from rockets and satellites. Results from an NRL rocket flight of a spectroheliograph and heliograph which gave $\sim 4$ " resolution have been described by Brueckner and Bartoe (1974). These observations show the behaviour of the lines in the supergranulation network, in active regions, coronal holes and sunspots. A preliminary discussion of the spatially resolved observations of the EUV lines obtained with the NRL instruments on ATM has been given by Tousey et al. (1973). Similarly the Harvard ATM instrument has been used to study a variety of features including the supergranulation network (Reeves et al., 1974). At around $5^{\prime \prime}$ resolution the observations in lines formed at $\sim 10^{5} \mathrm{~K}$ show that the boundary regions are about an order of magnitude brighter than the cell centres, but the intensity within the boundaries is by no means uniform. Using the observed upper limits to the boundary area $(<35 \%$ of the total) it is found that about $85 \%$ of the EUV emission originates from these regions. By $T_{\mathrm{e}} \sim 1.5 \times 10^{6} \mathrm{~K}$, where $\mathrm{Mg} \mathrm{X}$ is formed, the network is generally unrecognisable. Timothy et al. (unpublished) have recently suggested that there is evidence from the Harvard ATM observations that the average network intensity shows a long term decrease over the Skylab missions. This would clearly have an important bearing on the origin and formation of the supergranulation network. Bruns et al. (1975) have obtained observations of lines in the range 970-1400 $\AA$ with a stigmatic spectrograph on Salyut 4 . The spatial resolution was $\sim 5^{\prime \prime}$ and the spectra show enhancements associated with bright regions in the supergranulation network (also observed as flocculi). An interesting aspect is the obser- 
vation, in the bright-features on the disk, of the forbidden lines of Fe XII, usually observable only at the limb. The wings of $\mathrm{Ly}-\alpha$ are apparently very wide and have a shape that is not consistent with Doppler broadening. Further emission line widths have been obtained by Boland et al. (1975), which confirm and extend earlier conclusions, by these andother workers, that a non-thermal velocity component is present. The velocities are typically half the sound velocity ranging from $\sim 5 \mathrm{~km} \mathrm{~s}^{-1}$ at $10^{4} \mathrm{~K}$ to $25 \mathrm{~km} \mathrm{~s}^{-1}$ at $10^{5} \mathrm{~K}$. In a spherically symmetric model these would correspond to energy fluxes of $\sim 10^{5}-10^{6}$ ergs cm $\mathrm{cm}^{-2} \mathrm{~s}^{-1}$. Line widths have also been studied by Kohl et al. (1973 and 1975 (unpublished) and Feldman and Behring (1974) for $\mathrm{He} I$ and $\mathrm{He}$ II and a range of other ions). For ions formed in the temperature range $3 \times 10^{5} \mathrm{~K}-2 \times 10^{6} \mathrm{~K}$ these latter authors find velocities in the range $25-40 \mathrm{~km} \mathrm{~s}^{-1}$. Bruner et al. (1973) have given a preliminary report of line-width studies with sufficient resolution to show the supergranulation structure. Recent observations (Brueckner, unpublished) made by NRL using a high-resolution telescope and spectrograph, flown on a rocket, with in-flight pointing control, achieved a spectral resolution of $0.06 \AA$ at $1^{\prime \prime}$ spatial resolution. The spectra include the limb, quiet and active regions and a sunspot, and show a wide range of intensity and velocity variations.

Vernazza et al. (1975) have analysed the variations of the EUV line intensities with time and find a periodic, corelated brightness fluctuations through the chromosphere and transition region. Only the $\mathrm{H}$ Ly- $\alpha$ and $C$ II $(1335 \AA)$ lines show any evidence of a 300-s periodicity, and the lifetimes of the brightenings $(\sim 70 \mathrm{~s})$ are not consistent with those of spicules. Sentman and Shawhan (1974) have also reported negative results in a search for 300-s period oscillations at 2-cm wavelengths. Jakimiec and Jakimiec (1974) have observed periodic (200-900 s) variations in the $1-8 \AA$ solar X-ray flux, but this emission will originate from active regions.

The OSO-8 satellite, now operating, should provide valuable new information concerning the velocity fields and hopefully will improve our understanding of just which wave modes heat the different layers of atmosphere.

\section{B. The Transition Region in Other Solar Features}

\section{Active Regions}

Although a full account of work on active regions cannot be included in this report many of the observations and analyses discussed in the previous section relate to both quiet and active regions. Recent analyses support the conclusions of earlier work in which it was found that the transition region has a steeper temperature gradient in active regions than in the quiet sun (e.g., Dupree et al. (1973), Withbroe and Gurman (1973), Dubov and Kartoshova (1974), Billings and Alvarez (1975)). Work on models which account for the structure of quiet and active regions in terms of flux tubes (Landini and Monsignori-Fossi, 1975), Jordan (1975a) shows that the temperature gradient in the transition region increases as the maximum temperature in the flux tube increases.

\section{Prominences}

Prominences will be included in the report of Commission 10, and only work relating to the transition region and inner corona will be mentioned here. The EUV emission from prominences has been studied with the higher spatial resolution available from ATM. Schmahl et al. (1975) have used the Harvard ATM instrument to analyse spectroheliograms including quiescent prominences. They find that in the transition region around the prominence the density is 0.4 that in the normal transition region, whilst the temperature gradient is 1.4 steeper. The colour temperature of the Lyman continuum has a mean value of $6600 \mathrm{~K}$ and increases upwards in the prominence. The He I emission from prominences has been discussed by Heasley et al. (1974) and Morozhenko (1975). Chiuderi and Drago (1975) have shown that the presence of the transition region between a filament and the surrounding corona can be deduced from $6-\mathrm{cm}$ radio observations. The behaviour of eruptive prominences as observed with the NRL ATM spectroheliograph has been discussed by Tousey et al. (1975). 


\section{Sunspots}

The transition region directly above sunspots has been studied from the NRL rocket spectroheliograph observations (Brueckner and Bartoe, 1974), the Harvard instrument on ATM (Foukal et al., 1974), and the NRL rocket-bourne high-resolution telescope (Brueckner, unpublished). The results show that in the middle of the transition region $\left(T \sim 10^{5} \mathrm{~K}\right)$ the lines are enhanced by up to two orders of magnitude above quiet sun levels, and are brighter then in the surrounding plage region, whilst chromospheric and coronal lines are not enhanced as much. The electron density is lower directly over the spot than in the surrounding plage, and hence the temperature gradient must be at least an order of magnitude lower than in the plage region.

\section{Coronal Holes}

A discussion of the extended coronal aspect of these regions is given in the following section. The ATM observations, (Tousey et al., 1973; Bohlin et al., 1974; Huber et al., 1975) and recent rocket flights (Brueckner and Bartoe, 1974) have confirmed earlier findings from rocket and satellite experiments. In particular the greater thickness of the transition region in coronal holes has been directly observed from the width of the Ne VII emitting region in spectroheliograms. The holes are seen most clearly in lines formed at $T_{\mathrm{e}}>5 \times 10^{5} \mathrm{~K}$ but are in fact detectable in all layers above $10^{4} \mathrm{~K}$. The lines of $\mathrm{He} \mathrm{I}$ and $\mathrm{He}$ II which have an anomalously reduced emission in coronal holes, compared with other transition region lines, are discussed later.

\section{Transient Aspects of the Transition Region and Inner Corona}

\section{Macro-Spicules}

The ATM observations have shown the existence of a new class of EUV transient feature at the lines in polar coronal holes. These features appear to have the characteristics of small surges or giant spicules and hence have been called macro-spicules. They have been observed particularly clearly in the NRL ATM spectroheliograms made in the He II $304 \AA$ line (Bohlin et al., 1974, 1975). The macro-spicules reach heights of between 5-60" have diameters of $\sim 5-30 "$ and lifetimes of from 5 to $40 \mathrm{~min}$. They occur only within the network boundaries in coronal holes and their trajectories appear to be controlled by local magnetic fields. In the Harvard ATM data the macro-spicules have been observed in other transition region lines (Withbroe et al., 1975). Any model for their origin must explain why they occur only in coronal holes.

\section{Bright-Points}

Bright points may be considered as either an active or a quiet phenomenon. They were first observed in the X-ray region in spectroheliograms obtained from rockets flown by the American Science and Engineering Group (a.g. Vaiana $e t$ al., 1973), and have also been studied with the AS\&E instrument on ATM (Golub et al., 1974, 1975). Their lifetimes are $\sim 8 \mathrm{~h}$, their areas are $\sim 2 \times 10^{8} \mathrm{~km}^{2}$ distinct bi-polar structure, and the brightness increase they exhibit amount to several orders of magnitude within a few minutes. Their contribution to the overall conditions in the corona may be important since some 1500 are observed to occur per day. Whilst observed all over the Sun there is some evidence of a concentration towards active regions. Pikel'ner (1974) has suggested a link between visible region 'moustaches' and X-ray bright points.

\section{The He I and He II Lines}

The physics of the excitation of the helium lines and continua has been the subject of renewed lively discussions. Until the excitation processes are understood it is difficult to make further use of these lines in determining the atmospheric structure or helium abundance. Milkey 
et al. (1975) have made new calculations of the He I levels using a 13-level atom. According to their calculations and as found also in earlier analyses, the intensity of the He I continuum is dominated by the ultra-violet radiation from the corona, and recombination following this photo-ionization plays an important part in the population of the excited levels which give rise to the visible region lines. However, Milkey et al. conclude that the resonance line at $584 \AA$ is dominated by collisional excitation. But in order to explain the absolute intensity of the resonance line they have to invoke a temperature 'plateau' around $T_{\mathrm{e}}=4.5 \times 10^{4} \mathrm{~K}$. The anomalously high intensities of the He I and He II lines, when compared with other transition region lines formed at the same temperatures, were pointed out by Jordan (1975). It was proposed that these high intensities in the quiet sun could be accounted for if the resonance lines are formed by collisional excitation whilst the helium atoms and ions are in a state of transient ionization, such a process being made possible by the steep temperature gradient in the transition region. The great reduction of $\mathrm{He} \mathrm{I}$ and $\mathrm{He}$ II in coronal holes and lack of enhancement over sunspots can then be explained by the lower temperature gradients in these regions. Shine et al. (1975) have suggested that thermal and concentration diffusion across the steep temperature gradient could be the required enhancement process. Linsky et al. (1975) have analyzed the He II Lyman series lines and continuum observed from the OSO-7 satellite and also find that current models cannot account for the Ly- $\alpha(304 \AA)$ intensity. Zirin (1975) finds that the He I line at $10830 \AA$ is also reduced in polar regions and measures the normal height of the emission to be $\sim 2100 \mathrm{~km}$. He suggests that photo-ionization by coronal radiation is the controlling mechanism. Pope and Schoolman (1975) have also measured the height of the $\mathrm{D}_{3}$ line and find it to be $\sim 1350 \mathrm{~km}$, and similarly attribute its formation to the penetration of coronal EUV radiation. Milkey (1975) points out that Zirin's suggestion would imply strongly self-reversed line profiles for the $584-\AA$ and $304-\AA$ lines, and these are not observed. The widths of the He I and He II lines have been measured by several groups (Feldman and Behring, 1974; Doschek et al., 1974; Cushman et al., 1975; Delaboudiniere and Crifo, 1975). The relative importance of the collisional excitation and photo-ionization/recombination processes can be tested from observations of the He II B $\alpha$ multiplet at $1640 \AA$. Measurement of the width and shape of this blended multiplet (Kohl et al., 1973 and 1975 (unpublished), Boland et al., 1975, Feldman $e t$ al., 1975) suggest that a substantial fraction originates from collisional excitation, but Kohl et al. (unpublished) do observe narrow components which could be due to recombination.

\section{E. Diagnostics}

A full discussion of atomic data relevant to solar physics is not appropriate to this report (see Commission 14), but the following work is directly applicable to solar observations and in many cases has importance in methods of determining electron densities. Summers (1974) has made a fuller calculation of the density dependence of di-electronic recombination. Nussbaumer and Storey (1975) have investigated the effects of metastable levels and photo-ionization on the ionization equilibrium populations of $\mathrm{C} \mathrm{I}$ to $\mathrm{C} \mathrm{V}$. Excitation cross-sections and for transition probabilities of use in interpreting solar EUV spectra have been calculated by Mason (1975a) Fe X, XI, XIV, Ca XII, XIII, XV. Jackson (1973), Dankwort and Trefftz (1975) and Flower and Nussbaumer (1975a, 1975b) have made calculations for ions in the B I-like sequence. The latter authors also discuss the density sensitivity of lines of Si X and S XII. Calculations have been made for Fe XIII and Fe XII by Flower and Pineau des Forets (1973) and by Flower (1975), respectively, and again density sensitivities are discussed. From an analysis of quiet and active regions observed in the March 1970 total eclipse Gabriel and Jordan (1975) find that currently available atomic data for the EUV forbidden lines of Si VIII, Si IX, $\mathrm{Fe} \mathrm{XI}$ and Fe XII underestimate the populations of the upper levels of these transitions. Lines in the Be-I-like sequence also provide a means of measuring Ne. Cross-section calculations by Flower and Launay (1973) are in general agreement with earlier results, but when applied to solar data (Loulergue and Nussbaumer 1974) lead to very low values of $\mathrm{Ne}$, both for $\mathrm{C}$ III and $O \mathrm{~V}$. Jordan (1974) suggests that changes to the atomic data, within the quoted errorbars, are needed. Dupree et al. (1975, unpublished) have analysed C III spectra and spectroheliograms 
obtained with the Harvard ATM instrument and have discussed the electron density and its variation in a wide range of solar features.

\section{F. Abundances}

There has been little change over the past three years in values of abundances derived from observations of the transition region and corona. Current determinations are limited to about $\pm 50 \%$ accuracy by the combined uncertainties in intensity calibrations and atomic data. Apart from helium the least certain value for an abundant light element is that for neon. Values of between 4 and 21 have been reported for the oxygen to neon ratio (Acton et al., 1975; Walker et al., 1974; Malinovsky and Heroux, 1973), but there is no systematic difference between results for transition region and coronal lines.

\section{G. Line Identification}

Since atomic data lie within the scope of Commission 14 only a few new results will be mentioned. A review of lines identified in the solar spectrum (mainly below $800 \AA$ ) has been given by Fawcett (1974). An extensive list of lines observed in the region 160 to $770 \AA$ has resulted from recent flight of a 3-m grazing-incidence spectrograph (Behring et al., 1975). A review of intersystem and forbidden lines observed in the solar spectrum was given by Jordan (1973). New and revised identification for intersystem and forbidden lines of Fe IX in the visible and EUV spectrum have been proposed by Svensson $e$ t al. (1974). The intersystem lines were also identified from solar limb and disk spectra obtained by Firth et al. (1974). MagnantCrifo (1975) have discussed the intensities of the visible region forbidden lines of Fe IX. Noyes et al. (1975) have identified the Ne VI ground-state doublet to metastable quartet transitions for the first time, from sunspot spectra. The wavelengths agree well with those predicted by Edlen et al. (1969).

\section{THE OUTER CORONA \\ (R. M. Mac Queen)}

\section{A. Coronal Density and Temperature}

Photometric and polarimetric observations in white light during total solar eclipses continue to be the chief source of estimates of the line-of-sight electron density in the corona, both background and for discrete forms; results include those from the eclipses of 1922, September 20 (Eddy, 1973); 1952, February 25 (Durst, 1973); 1961, February 15 (Dollfus et al., 1974); 1966, November 12 (Saito and Tandberg-Hanssen, 1973; Kirk and Newby, 1974); 1968, September 22 (Vasil'ev, 1973); 1970, March 7 (Waldmeier and Weber, 1974; Bappu et al., 1973; Waldmeier, 1975a; Koutchmy and Magnent, 1973; Sazanov, 1973; Orrall and Speer, 1973); 1972, July 10 (Koutchmy et al., 1974; Bareau, 1973; Bohlin and Garrison, 1974; Poulain, 1974); 1973, June 30 (Koutchmy et al., 1973; Poulain, 1974; Waldmeier, 1974; Durst, 1975; Keller, 1975; Sykora, 1975; Rusin and Rybansky, 1975); and 1974, June 20 (Waldmeier, 1975b). Koutchmy and Koutchmy (1973) examined the coronal aureole from results obtained at the eclipses of 1968, September 22 and 1970, March 7, and Rozelot and Ratier (1975) studied telluric scattering near the solar limb at the partial eclipse of 1971 February 25 . Long term synoptic studies of the corona and related areas include those of: Leblanc et al. (1973), who find that, even at times of relatively high solar activity, the density of selected quiet regions nearly approximate the model of Saito for a minimum corona; Leroy and Trellis (1974a), who find a nearly linear relation between the K-coronal integrated intensity and the Wolf sunspot number. The behaviour of the $5303 \AA$ line was different, with relatively higher intensities before the end of the cycle; Antonucci and Duvall (1974), who examined variations over a solar cycle of the Fe XIV 5303 \& emission; Antonucci and Svalgaard (1974) who examined the correlation between Fe XIV $5303 \AA$ and sector structure; Iucci et al. (1973) who have studied the modulation of cosmic rays and the correlation between the radar cross-section 
of the corona and solar wind velocity. Leroy and Trellis (1974b) have computed densities and temperatures of the lower corona and find it necessary to interpret the results in terms of inhomogeneities in the emission; the degree of inhomogeneity was found to be solar cycle dependent. Additional measurements of the inhomogeneous nature of the lower corona have been examined by Fort et al. (1973), employing results of Picat et al. (1973) on the 5303 and $6374 \AA$ emission from individual structures in the low corona. Also, Nöens and Rozelot (1974) and Rozelot and Nöens (1975) have examined coronagraph observations of the $7892 \AA$ line of $\mathrm{Fe} \mathrm{XI}$, and in combination with $5303 \AA$ observations have determined the intensity and density gradient in a coronal structure. Leroy et al. (1973) have examined temperatures deduced from measured density gradients in the corona and the assumption of hydrostatic equilibrium, and those deduced from line ratios, but find no clear relationship or trend. Howard and Koomen (1975) have examined variations in the total $(K+F)$ brightness of the white-light corona observed over a 15-month period (1971-1972) with the OSO-7 coronagraph (Koomen et al., 1975), and show them to be correlated with general activity as measured by the Zurich sunspot number.

A new, potentially accurate, method of determination of the density of the outer solar corona has been introduced (Counselman and Rankin, 1972; 1973), based upon measurements of the dispersion of pulsar signals. These show that with a decrease in activity from 1969 to 1971 , the corona became more concentrated toward equatorial regions, although the density remained virtually constant ( 8000 electrons $\mathrm{cm}^{-3}$ at $\left.10 R_{\odot}\right)$. Also, a decrease in multipath scattering delay was interpreted as due to a marked decrease in coronal inhomogeneity in 1971 as compared to 1969 and 1970. Perry and Altschuler (1973) have described improved numerical techniques for deducing the three-dimensional electron density distribution in the global corona.

Observations of visible region line emission from the corona for the purpose of temperature determinations have been analyzed for eclipses of 1965, May 30 (Liebenberg et al., 1975; Magnant-Crifo, 1973, 1974, 1975) and 1970, March 7 (Marshall and Henderson, 1973).

The coronal temperature and temperature gradient at $\sim 3 R_{\odot}$ have been studied by Bame $e t a l$. (1974) through examination of the relative abundances of iron ions in the solar wind, and the temperature at $1.5 R_{\odot}$ through composite abundances of other ions. The values so determined, despite considerable spread, are in close agreement with earlier coronal line-width measurements at the lower heights (cf. Billings, 1974). Some simplified theoretical considerations of the distribution of temperature in open and closed structures in the corona have been examined by Vil'koviskii (1973). Athay (1973) finds a distinct difference between the coronal density and temperature gradients depending on whether or not the field configuration is open or closed, with higher density gradients in open field regions and vice-versa. Rusin (1973) has observed differences in the $5303 \AA$ intensity gradients which depend on the solar activity present.

\section{B. Coronal Structures}

The conditions in coronal streamers have been examined by Eddy (1973), Saito and Tandberg-Hanssen (1973), Waldmeier and Weber (1974), Durst (1974), Dollfus et al. (1974) and Liebenberg et al. (1975) from eclipse observations. Bohlin and Garrison (1974) have computed a three dimensional model for streamers observed at the 1972, July 10 eclipse and from rocket coronagraph observations. The utility of type III bursts as indicators of the density structure of streamers remains unclear (Mercier, 1973; Boischot, 1974; Bradford and Hughes, 1974; Caroubalos et al., 1974; Leblanc et al., 1974; Rosenberg, 1975; Pick et al., 1975); Fort and Matres (1974) have examined the geometrical structure of the quiet corona in the region of quiescent prominences. They find the lower coronal material apparently has a planar geometry similar to that of the prominence, and, in fact, lies in the same plane as the prominence. The conditions in coronal condensations have been investigated by Fort et al. (1973), Vasil'ev (1973), Kurokawa (1975) and Mason (1975b), the latter employing new emission rates for forbidden lines (Mason, 1975a), and in the microwave region by Tanaka and Énomé (1975). Magnant-Crifo (1974) has examined abundances and the material distribution as functions of 
density and temperature for a condensation observed at the 1965, May 30 eclipse. And Tetrusshvili (1974) has examined the Ca XV 5694 emission present in several structures in 1971 and 1972 and has deduced their electron densities. Vaiana et al. (1973) have summarized the results of a series of rocket observations in the soft X-ray spectral region, and categorized the array of structures observed. These authors also summarized techniques for reduction of broadband soft X-ray observations. Vaiana $e t$ al. (1973) have presented initial results on the evolution of X-ray structures from Skylab-ATM observations.

The relationship of a well-defined loop prominence system observed simultaneously in $\mathrm{H} \alpha$ and $5303 \AA$ has been examined by McCabe (1973) who finds a two-dimensional spatial separation between the hot and cool material.

Coronal holes receive increasing emphasis as their possible relationship to the solar wind continues to be explored. They are recognized principally on the basis of their signature in the EUV and X-ray spectral range - regions of dramatically low emission measure. On the basis of results from rocket observations of 1970, March 7 and November 24, Krieger et al. (1973) pointed out the association between a coronal hole and a recurrent high velocity stream in the solar wind. Neupert and Pizzo (1974) and Bell and Noci (1975) obtained similar results through a comparison of holes identified in the EUV from the OSO-7 satellite, geomagnetic indices, and in the latter study, solar wind velocity. Krieger et al. (1974), employing Skylab-ATM and IMP-H results, have investigated more thoroughly the conditions of coronal hole topology leading to distinct associations with high-speed streams. Estimates of the emissivity and electron density in a hole have been computed by Fisher and Musman (1975) on the basis of limb measurements of Fe XIV $5303 \AA$. Pneuman (1973) has pointed out the suitability of conditions within a coronal hole for enhanced flow of coronal material, and the relative roles of mechanical, conductive and radiative fluxes in coronal holes have been examined by Noci (1973). In addition, Durney and Hundhausen (1974) have computed the expansion of the solar wind from low-density regions such as thought to be present in holes, and included the effects of modified thermal conduction due to the spiral field structure. Waldmeier (1975a) has examined the boundary of the coronal hole present near the west limb of the Sun at the time of the 1970, March 7 eclipse, and has estimated the electron density of the central region of the hole. Coronal holes can be identified, and a temperature and density determined, from metric radio emission (Dulk and Sheridan, 1974; Lantos and Avignon, 1975). The latter study extends over two cycles and indicates conditions in the holes do not vary systematically with the cycle of activity. Furst and Hirth (1975) have also detected holes at $2.8 \mathrm{~cm}$. The rotation rate of coronal holes as observed in soft X-rays from the Skylab-ATM has been examined by Timothy et al. (1975); they find that rates commensurate with rigid rotation are apparently appropriate for holes; a result which has been confirmed by Wagner (1975), utilizing the absence of Fe XV $284 \AA$ emission in coronal holes. The rigid rotation of coronal features has also been discussed by Antonucci (1975).

Finally, the abundance and surface distribution of polar plumes at the 1966, November 12 eclipse have been examined by Kirk and Newby (1974); their work is in substantive agreement with that anticipated by Saito's earlier studies.

\section{Coronal Magnetic Fields}

The specification of the coronal magnetic field direction and strength remains within the domain of computations of the extension of photospheric magnetic fields under certain limiting assumptions. Principally, there are the assumptions of a potential (current-free) configuration, and the requirement that the photospheric source of the fields remain constant over successive limb passages. Levine and Altschuler (1974) have examined the relaxation of the first assumption, and have determined that relatively large electric currents are required before significant topological deviations from the potential magnetic-field configuration can be noticed. The identification of stable, long-lived outer coronal structures by satellite observations (OSO 7, Skylab-ATM) will provide the selection necessary to meet the second principal assumption of the computations, and thus permit detailed examinations of the role of potential fields in the corona. 
The large-scale coronal magnetic field has been examined through models of the calculated potential field (Newkirk et al., 1973; Altschuler et al., 1975); the relationship between the coronal magnetic field and the interplanetary sector structure has been studied by several workers. Wilcox and Svalgaard (1974) compared computed coronal potential fields and observations of interplanetary sector structure, and suggested that an extended magnetic arcade loop structure was the coronal signature of the sector boundary. Svalgaard et al. (1974) extended this association to account for the presence of polar magnetic field regions in a phenomenological model. Consistent with the general features of this model are observations of recurrent inner coronal structures (Hansen et al., 1973) and, in the outer corona, recurrent structures observed from the OSO-7 satellite (Howard and Koomen, 1974). The various workers do not agree, however, on the details of the orientation of the mapping of the interplanetary sector structure to the Sun. Eddy et al. (1973), from eclipse polarization measurements, were able to place upper limits on the magnitude of non-radial magnetic-field components in the corona at their observation time. Satellite and rocket observations of the EUV and X-ray emission from low coronal structures now permit detailed examination of the configuration of closed magnetic fields - loops. Observations of the Fe XV and Fe XVI emission from one such loop by the OSO-7 spectroheliograph (Neupert et al., 1974) have been compared with computations of non-potential arches (Neupert et al., 1975). The observed arches were seen to change orientation with height in respect to the underlying magnetic neutral line; this was interpreted as an indication of differing electric currents along ivarious field lines. A source of such coronal currents has been examined by Hevaerts (1974); he suggests that small photospheric motions are adequate to drive $\sim 10^{-1} \mathrm{~A} \mathrm{~m}^{-2}$ vertical current through coronal lines of force connecting separate photospheric regions. Molodensky (1974) has examined the conditions of equilibrium and stability of force-free magnetic fields. In the future, the wealth of well-resolved observations of coronal features now available from rocket and satellite experiments will provide a fruitful field for investigation of the small to medium scala magnetic field topology.

\section{Coronal Heating}

Various workers have continued to investigate the origin of the heating of the solar atmosphere. Piddington (1973) has examined various modes of mechanical waves; accoustic, Alfvén and gravitational, and suggests a model for Alfvén wave heating. Uchida and Kaburaki (1974) examined heating by the decay of slow-mode MHD waves from the photosphere or subphotospheric layers. Wentzel (1974) has computed the temperature rise of regions heated by Alfvén waves, a rise which he found is independent of electron density and wave intensity, and independent upon magnetic pressure and wave period. On the other hand, Levine (1974b) and Tucker (1973) have suggested rather different approaches to heating mechanisms. In the former work, it is suggested that magnetic fields in the low-corona collapse and accelerate particles to a few times thermal velocity (Levine, 1974a); this energy is then available to the corona through Coulomb collisions. Tucker (1973) has suggested that solar active regions are heated by the relaxation of stressed magnetic-field configurations, presumably in current sheets.

Nakagawa et al. (1973) have examined the conditions for existence of trapped waves in the lower solar atmosphere; these may be of relevance, for example, in the boundary of supergranulation cells, plages, and sunspots. Finally, heating in the outer corona due to hydromagnetic wave dissipation has been examined by Hung and Barnes $(1973 a, b, c)$ in the cases of varying conditions of mutual interaction of electrons or protons.

\section{E. Coronal Expansion and Flow}

The dynamics of coronal material flow has been examined by several workers. Browne and Bessey (1973) have examined a time-dependent model of the hydrodynamic equations of the coronal gas extending from the transition region outward, and deduced a 'settling time' into dynamic equilibrium. Knight et al. (1974) analyzed a simple two-fluid model of the coronal medium, and found that electron and proton temperatures may differ significantly. Pneuman (1973) examined the influence of the solar wind on large-scale temperature and density distri- 
butions in the corona. Durney and Pneuman (1975) have presented results of a three-dimensional non-radial model of solar wind expansion (to $1 \mathrm{AU}$ ), employing boundary conditions based upon potential field models, and observations of the inner corona near the time of the 1966, November 12 eclipse. They found important discrepancies between the model predictions and observations at $1 \mathrm{AU}$, which they attributed to two causes - the rapid divergence of the coronal magnetic-field model, and the breakdown in the validity of the heat conduction model close to the Sun. An isothermal model of the solar wind equations, employing a latitude variation of coronal parameters has been attempted by Roxburgh and Singer (1975); the results were found to be dependent on the geometry of the injection angle of the fluid at the coronal base, and not uniquely determined by the base density. Gussenhoven and Carovillano (1973) have investigated the validity of hydromagnetic equations in the case of the introduction of the polar angle, and find inconsistencies for radial flows and fields. Finally, Priest and Pneuman (1974) have examined the role of non-spherically symmetric magnetic fields on the overall loss of angular momentum from the Sun, and found, upon accounting for the coupling between the solar wind and magnetic field explicitly, the angular momentum loss is substantially reduced as compared to previous estimates employing monopole fields.

The theory of particular structures in the corona have been investigated by: Durney (1975), who considered streamers with $T$-type neutral points, and the stability of the hydrostatic equilibrium condition to the relative magnitudes of magnetic flux of open and closed configurations; Weber and Rosenberg (1974), who have examined a model of a stationary isothermal streamer proposed by Pneuman and discussed a procedure for determining model parameters from type III radio burst information; Raadu and Kuperus (1973), who have studied the formation of quiescent prominences from thermal instabilities of current sheets in the corona; and by Guseinov (1973), who investigated the condensation of the coronal gas.

\section{F. Low Excitation Emissions in the Corona}

In an attempt to further study the origin of the presence of coronal lowexcitation line emissions (e.g., $\mathrm{H} \alpha, \mathrm{D}_{3}, \mathrm{H}$ and $\mathrm{K}$ ), Stellmacher and Koutchmy (1974) have examined results, from the 1973, June 30 eclipse, which effects of $\mathrm{Ca}^{+}, \mathrm{H}, \mathrm{K}, \mathrm{H} \delta, \mathrm{H} \gamma$, and $\mathrm{H} \beta$. They concluded the emission arises from double scattered light of chromospheric origin in the Earth's atmosphere. On the other hand, Alikayeva (1975) has observed a discrete coronal region whose structure is evident in a number of lowexcitation lines. Beckers and Chipman (1974) have examined the profile and polarization of the coronal Ly- $\alpha$ line - which arises from chromospheric $L y-\alpha$ scattering on neutral $\mathbf{H}$ in the corona. Because of its relatively low atomic weight, the line is a sensitive indicator of the coronal temperature, and they investigated effects of the coronal expansion and turbulence on the line visibility.

\section{G. Emission Line Polarization}

A non-LTE formulation of the transfer of polarized radiation, in the presence of a magnetic field, has been completed, and applied to coronal lines (House and Steinitz, 1974, 1975). Particularly, the polarization of the emission line Fe XIV $5303 \AA$ has been computed for magnetic-field geometries specified by potential coronal field models (House, 1974). Crosssections for proton and electron excitation in Fe XIII and Fe XIV have been examined in some detail by Landman (1973), Finn and Landman (1973), Sahal-Brechot (1974a, b) and Flower and Pineau des Forats (1973), and employed in the above calculations.

Initial out-of-eclipse observations of the Fe XIV $5303 \AA$ emission showed a linear polarization of up to $15 \%$ (near $1.3 R_{\odot}$ ), deviating only slightly from the radial direction, and increasing with increasing height (Mickey, 1975) have failed to detect measurable polarization in Fe XIV $5303 \AA$ to heights of $1.4-1.5 R_{\odot}$. However, Mogilevsky et al. (1973) have reported polarizations in excess of $30 \%$ from observations during the 1970, March 7 eclipse. During the 1973 , June 30 eclipse Sykora et al. (1975) measured polarizations in $5303 \AA$ of between $10 \%$ and $35 \%$. Airborne observations of the Fe XIII $10747 \AA$ emission line polarization at the eclipse of 1966, November 12 (Eddy et al., 1973) indicate a steep gradient of polarization, varying 
from near zero at the limb to higher than $80 \%$ at $1.6 R_{\Theta}$ and indicate qualitative agreement with computations on the relative role of collisions in the corona for Fe XIII.

\section{H. Anomalous Coronal Continuum Polarization}

Sazanov (1973) has analyzed polarimetric photography of the inner corona at the 1970 , March 7 eclipse and has found percentage polarizations which substantially exceed (1.3-1.5 times) the maximum theoretical value; the polarization direction in these regions is consistent with that predicted by theory. Molodensky (1973) has attempted to explain these and earlier observations of 'anomalous polarization' as due to bulk streams of fast electrons moving in preferential directions in the corona.

\section{Fraunhofer Corona}

The F-corona, usually regarded as an impediment to studies of the electron-scattered coronal component, is itself of great physical interest. Mukai et al. (1974) and Lamy (1974a) have reexamined the dynamics of dust particles near the Sun and refined earlier work. Observations of the intermediate infra-red $(\sim 10 \mu)$ emission at the 1970, March 7 and 1973, June 30 eclipses were directed toward identification of the spectral character of the infra-red emission from the near-solar material. The former measurements (Mankin et al., 1974) could only place upper limits on the intermediate infra-red emission, which was shown to be consistent with earlier models of silicate emission. The latter observations (Lena et al., 1974a, b) made from the 'Concorde' aircraft were able to discern spectral features consistent with the 'reststrahlen' band of silicate (e.g. Knacke and Thomson, 1973). These observations seem to rule out the possibility of a major contribution by graphite (Mukai and Mukai, 1973), and are consistent with the predictions of Lamy (1974b) who has reexamined the interaction of the F-coronal grains with the solar radiation field. In addition, Lamy and Koutchmy (1975) have investigated the spatial structure of the $2.2 \mu$ infra-red emission at the same eclipse. At the opposite end of the spectrum, Orrall and Speer (1973) have examined the continuum coronal radiation at wavelengths below $2220 \AA$, obtained by rocket at the 1970 , March 7 eclipse, in an attempt to determine the ultraviolet scattering efficiency of the F-coronal material, for comparison with the observed behaviour of the zodiacal light. Koutchmy and Magnant (1973) have reexamined the separation of the visible F- and K-coronal components, employing results from the 1970 , March 7 eclipse; they find the measurements are substantially in agreement with previous determinations, and Sanford et al. (1973) have examined the F-coronal contribution during the 1972 , July 10 eclipse.

Abbreviations

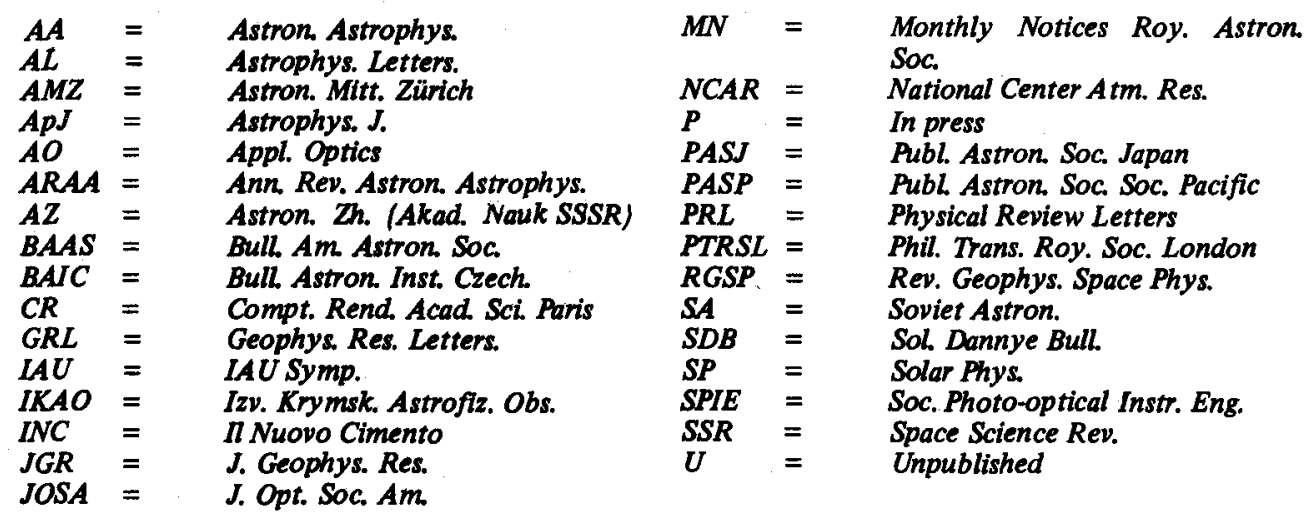




\section{REFERENCES}

Ackerman et al.: 1973, SP 30, 345.

Acton, Catura and Joki: $1975, A p J 195, \mathrm{~L} 93$.

Aime and Ricort: 1975, $A A$ 39, 319.

Alikayeva: 1975, $S P 41,89$.

Alissandrakis: 1973, SP 32345.

Allen and Cowley: 1974, AA 36, 315.

Allen and Hall: 1973, BAAS 5, 445.

Aller: 1973, PASP 85, 529.

Altrock: 1974, SP 34, 37.

Altrock and Canfield: $1974 \mathrm{ApJ}$ 194, 733.

Altrock and Cannon: 1972, SP 26, 21.

Altrock and Cannon: 1973, SP 20, 31.

-Altschuler et al.: 1974, SP 39, 3.

Altschuler et al. : 1975, SP 41, 225.

Anderson and Sorensen: 1974, SP 38, 343.

Antonucci: 1975, INC 25, 513.

Antonucci and Duvall: 1974, SP 38, 439.

Antonucci and Svalgaard: 1974, SP 36, 115.

Assousa and Smith: 1972, $A p J$ 176, 259.

Athay: 1973, SP 29, 357.

Athay: $1975, I A U 56,23$.

Athay and Lites: 1972, ApJ 176, 809.

Auvergne et al., 1973, $A A$ 29, 93.

Ayres: $1975, A p J(\mathrm{P})$.

Ayres and Linsky: 1975, ApJ (P).

Bame et al.: 1974, SP 35, 137.

Banos: 1973, SP 32, 337.

Bappu and Sivaraman: 1971, SP 17, 316.

Bappu, Bhattacharyya, and Sivaraman: 1973,

Pramana 1, 117.

Bareau: 1973, $A A$ 26, 467.

Bates and Mc Dowell: 1972, SP 23, 26.

Beckers: 1970, $A O$ 9, 595.

Beckers: 1974, SP 37, 351.

Beckers: 1975, ApJ 195, L43.

Beckers and Artzner: 1974, SP 37, 309.

Beckers and Chipman: 1974, SP 34, 151.

Beckers and Milkey: 1975, (P).

Beckers and Wagner: 1970, $A O 9,1933$.

Beckers, Dickson and Joyce: $1975, A O 14$,

Beckers, Dickson, Woodman: 1975, Opt. Enging.

14,64 .

Behring et al.: 1975, ApJ (P).

Bell: 1975, RGSP 13.

Bell and Nocci: 1975, JGR (P).

Benner et al.: 1972, Arizona NASA Atlas of the IR Solar Spectrum Report.

Berger and Lena: 1972 $A A$ 20,111.

Berthomieu: 1974, SP 38, 311.

Biemont: 1973, SP 32, 117.

Biemont: 1974 a, $S P$ 38, 15.

Biemont: 1974b SP 39, 305.

Billings: 1974, SP 38, 181.

Billings and Alvarez: 1975, SP 40, 23.

Blackwell et al. : 1972, $M N$ 160, 189.

Blackwell et al. : 1972, MN 160, 121.

Bohlin and Garrison: 1974, SP 38, 165.

Bohlin, Sheeley and Tousey: 1974, Space Res. XV (P).
Boland et al.: 1973, $A A$ 22, 161.

Boland et al.: 1975, MN 171, 697.

Bohlin et al. : 1975, BAAS 7, 354.

Bohlin et al: : 1975, ApJ 197, L133.

Boischot: 1974, AA 33, 379.

Boland et al. : 1973, AA 22, 161.

Boland et al. : 1975, $M N$ 171, 697.

Bonnet: 1975, Phil. Trans. Roy. Soc. (P).

Born: 1974, SP 38, 127.

Bradford and Hughes: 1974, AA 31, 419.

Brandt and Nesis: 1973, SP 31, 75.

Brault et al. : 1975, SP 41, 43.

Bray: 1973, SP 29, 317.

Bray et al.: 1974, SP 39, 323.

Brown et al: : 1974, JOSA 64, 1665.

Browne and Bessey: 1973, SP 31, 351.

Brueckner and Nicolas: 1973, SP 29, 301.

Brueckner and Nicolas: 1973, BAAS 4, 378.

Bruner et al.: 1973, $A p J$ 182, L33.

Bruns et al. : 1975, Space Res. XVI (P).

Burton et al. : 1973, AA 27, 101.

Cacciani and Fofi: 1971, SP 19, 270.

Caccin et al. : 1973, SP 33, 49.

Caccin et al.: 1974a, SP 35, 31.

Caccin et al.: 1974b, SP 35, 41.

Canfield: 1973, $A p J$ 179, 643.

Canfield and Mehltretter: 1973, SP 33, 33.

Canfield and Musman: 1973, BAAS 5, 269.

Caroubalos et al.: 1974, AA 32, 255.

Chapman: 1972, SP 24, 288.

Chapman: 1974, ApJ 191, 255.

Chapman and Ingersoll: 1972, ApJ 175, 819.

Chapman and Ingersoll: 1973, ApJ 183, 1005.

Cha and White: 1973, SP 31, 23.

Chen: 1974, SP 37, 53.

Chen and Lykoudis: 1972, SP 25, 380.

Chiuderi and Giovanardi: 1975, $S P 41,35$.

Chiuderi and Riani: 1974, SP 34, 113.

Chmielewski et al.: 1975, $A A$ 42, 1.

Cimino, Cacciani and Fofi: 1970, SP 11, 319.

Cocke et al.: 1973, ApJ 184, 653.

Councilman and Rankin: 1972, ApJ 175, 843.

Councilman and Rankin: 1973, $A p J$ 185, 357.

Cram: 1975, SP 37, 75 .

Culhane and Action: 1974 ARAA 12, 359.

Cushman: 1975, JGR 80, 482.

Dall'Oglio et al.: 1973, IR Phys. 13, 1.

Dall'Oglio et al.: 1974, IR Phys. 14, 327.

Dankwort and Trefftz: $1975 A A(\mathrm{P})$.

Day: 1974, SP 36, 25.

Debarbat et al.: 1970, AA 8, 231.

De Jager: 1972, SP 25, 71.

De Jager: 1974, $S P$ 34, 91 .

De Jager and Neven: 1972, SP 25, 277.

Delaboudiniere and Crifo: 1975, Space Res. XVI (P).

Delbouille et al: : 1976, Photometric Atlas of the 
Solar Spectrum from d 8000 to $\mathrm{d} 10000$.

Delone et al.: 1973, SA 17, No. 4.

Den: 1974, Soln. Dann. 12, 91.

Deridder and Van-Rensbergen: 1974, SP 34, 77.

Deubner: 1974, SP 36, 299.

Dicke: 1972, ApJ 175, 831.

Dicke: $1972, A p J 176,479$.

Dicke: 1973, $A p J$ 180, 293.

Dicke: 1974, $A p J 190,187$.

Dicke: 1974, SP 37, 271.

Dicke and Goldenberg: 1974, ApJ Sup. 27, 131.

Dolffus: 1974, Planets, Stars and Nebulae Studies with Photopolarimetry Tucson, p. 695.

Dollfus et al.: 1974, SP 37, 367.

Domke and Staude: 1973a, SP 31, 279.

Domke and Staude: 1973b, SP 31, 291.

Doscher, Behring and Feldman: 1974, ApJ 190 L141.

Drake: 1974, ApJ 189, 161.

Dravins: 1975, SP 40, 53.

Dubov: 1973, $A Z$ 50, 1333.

Dubov: 1974, $A Z$ 51, 671.

Dubov and Kartoshova: 1973, IKAO 471,49.

Dubov and Kartoshova: 1974, IKAO 52, 113.

Dulk and Sheridan: 1974, SP 36, 191.

Dumont and Pecker: 1971, $A A$ 10, 118.

Dumont et al.: 1973, SP 28, 271.

Dumont et al.: 1975, (P).

Dunn, Rust and Spence: 1974 Proc. SPIE 44, 109.

Dunn and Zirker: 1973, SP 33, 281.

Dupree et al.: 1973, ApJ 182, 321.

Durney: 1975, SP 41, 233.

Durney and Hundhausen: 1974, JGR 79, 3711.

Durney and Pneuman: 1975, SP 40, 461.

Durst: 1973, AMZ 320.

Durst: 1975, AMZ 335.

Eddy: 1973, SP 30, 385.

Eddy et al.: 1973, SP 30, 351.

Edlen et al.: 1969, SP 9, 432.

Edmonds: 1974, SP 38, 33.

Ekeland and Hauge: 1975, SP 42, 17.

Elste et al.: 1973, BAAS 5, 20.

Engvold and Hauge: 1974, Inst. Theor. Astr. Blindern Oslo 39.

Evans and Catalano: 1972, SP 27, 299.

Falciani et al. : 1974, SP 35, 277.

Fawcett: 1974, Advances Atomic Molecular Phys. 10, 223.

Feldman et al. : 1975, ApJ 199, L67.

Finn and Jefferies: 1974, SP 34, 57.

Finn and Landman: 1973, SP 30, 381.

Firth et al.: 1974, $M N 166,543$.

Fisher and Musman: 1975, ApJ 195, 801.

Flower: 1975, $A A(\mathrm{P})$.

Flower and Nussbaumer: 1975a, $A A(\mathrm{P})$.

Flower and Nussbaumer: 1975b $A A(\mathrm{P})$.

Flower and Pineau Des Forets: 1973, $A A$ 24, 181.

Flower and Pineau Des Forets: 1974, $A A$ 32, 297.

Fort et al.: 1973, $A A$ 24, 267.
Fort and Matres: 1974, $A A$ 33, 249.

Fossat and Roddier: 1971, SP 18, 204.

Fossat et al.: 1974, ApJ 193, 497.

Foukal: 1974, SP 37, 317.

Foy: 1975, $A A$ 39, 2.

Frazier: 1974, BAAS 6, 287.

Frezier and Stenflo: 1972, $S P 27,330$.

Fredga and Hogbom: 1971, SP 20, 204.

Frish: 1975, $A A$ 40, 267.

Furst and Hirth: 1975, SP 42, 157.

Gabriel: 1974, IAU 56, 295.

Gabriel: 1975, PTRSL (P).

Gabriel and Jordan: 1975, $M N(\mathrm{P})$.

Gebbie and Steinitz: 1974, $A p J$ 188, 399.

Gehren: 1975, $A A$ 38, 289.

Gezari et al.: 1973, AA 26, 409.

Gilman: 1974, ARAA 12, 47.

Giovanelli: 1974, SP 37, 301.

Giovanelli: 1975a, $S P(P)$.

Giovanelli: 1975b, SP (P).

Goldman et al.: 1973, ApJ 182, 581.

Golub et al.: 1974, ApJ 189, L93.

Golub, Krieger and Vaiana: 1975, SP 42, 131.

Gopasyuk and Kalman: 1972, IKAO 44, 52.

Gopasyuk and Tsap: 1972, SAAJ 16, 5.

Gopasyuk and Tsap: 1972, IKAO 45, 3.

Gopasyuk et al.: 1973, SP 31, 307.

Gouttebroze and Lemaire: 1974, $A A$ 34, 375.

Greve and McKeith: 1974, SP 37, 3.

Grevesse and Sauval: 1973, $A A$ 27, 29.

Grossman-Doerth, Kneer and Uexkull: 1974, $S P$ $37,85$.

Gurtovenko et al: : 1974, SP 37, 43.

Guseinov: 1973, SP 28, 457.

Gussenhoven and Carovillano: 1973, SP 29, 233.

Hall: 1973, ApJ 182, 977.

Hall: 1975, $A p J 197,509$.

Hall and Engvold: 1975, ApJ 197, 513.

Hammerschlag: 1973, JOSO Ann. Report, p. 85.

Hammerschlag: 1975, $A O$ 14, 885.

Hammerschlag and Zwaan: 1973a PASP 85, 468.

Hammerschlag and Zwaan: 1973b, JOSO Ann.

Report, p. 61.

Hansen et al.: 1974, GRL 1, 13.

Hart: 1974, ApJ 187, 393.

Harvey: 1973, SP, 28, 9.

Harvey and Schwarzchild: 1975, ApJ 196, 221.

Hauge: 1972a, $S P$ 26, 263.

Hauge: 1972b SP 26, 276.

Hauge: 1972c, SP 27, 286.

Hauge: 1974, SP 34, 33.

Hauge and S $\phi r l i: 1973, S P$ 30, 301.

Hauge and Youssef: 1975, SP 41, 67.

Hearn and Holt: 1973, $A A$ 23, 347.

Heasley, Mihalas and Poland: 1974, ApJ 192, 181.

Heath: 1973, JGR 78, 2779.

Hendl: 1974, BAAS 5, 288.

Heroux, Swirbalus and Cohen: 1975, JGR (P).

Heyvaerts: 1974, SP 38, 419. 
High Altitude Observatory: 1974a, Flare produced Shock Waves in the Corona and in Interplanet. ary Space, NCAR, Boulder.

High Altitude Observatory: 1974b, Flare-Related Magnetic Field Dynamics, NCAR, Boulder.

Hill et al. : 1974, PRL 33, 1497.

Hirshberg: 1973, RG SP 11, 115.

Holt: 1974, $A A$ 30, 185.

Holweger: 1972, SP 25, 14.

Holweger: 1973, AA 26, 275.

Holweger and Müller: 1974, SP 39, 19.

House: 1974, PASP 86, 490.

House and Steinitz: 1973, $A L 15,129$.

House and Steinitz: 1975, ApJ 195, 235.

House and Steinitz: 1975, $A p J(\mathrm{P})$.

Howard: 1973, BAAS 5, 273.

Howard: 1974, SP 39, 275.

Howard and Kooman: 1974, SP 37, 469.

Howard and Kooman: 1975, BAAS 7, 355.

Howard et al.: 1975, Report UAG-48 World Dato Center A, NOAA, Boulder.

Huber and Tubbs: $1973, A p J 186,1053$.

Huber et al. : 1975, ApJ 194, L115.

Hudson and Lindsey: $1974, A p J 187, \mathrm{~L} 35$.

Hung and Barnes: 1973a, ApJ 180, 253.

Hung and Barnes: 1973b, ApJ 180, 271.

Hung and Barnes: $1973 c, A p J 181,183$.

Illing et al. : 1975, BAAS 7, 363.

Ingersoll and Chapman: 1975, SP 42, 279.

Iucci et al. : 1973, SP 33, 505.

Jackson: 1973, J. Phys. B 6, 2325.

Jager: 1972, SP 27, 481.

Jager: 1974, SP 39, 499.

Jakimiec and Jakimiec: 1974, AA 34, 415.

Jefferies and Mc Allister: 1973, BAAS 5, 276.

Jones: 1973, BAAS 5, 274.

Jordan: 1973, Nucl. Instr. Methods 110, 373.

Jordan: 1974, AA 34, 69.

Jordan: 1975a, PTRSL (P).

Jordan: $1975 b, M N 170,429$.

Jordan and Ridgeley: 1974, MN 168, 533.

Joshpa et al. : 1973, SP 29, 385.

Kandel and Keil: 1973, SP 33, 3.

Kane: 1975, IAU 68.

Kanno and Tanaka: 1975, SP (P).

Kastner, Tothe and Neupert: 1974, AA 37, 339.

Keller: 1975, $U$.

Kiepenheuer and Mehltretter: 1964, AO 3, 1359.

Kirk and Newby: 1974, SP 35, 377.

Knacke and Thomson: 1973, PASP 85, 341.

Knight et al. : 1974, SP 37, 183.

Knoska: 1975, BAIC 26, 51.

Kohl and Parkinson: 1973, ApJ 184, 641.

Kohl, Parkinson and Reeves: 1973, BAAS 5, 274.

Kohl, Parkinson and Reeves: 1975, SSR (P).

Koomen et al. : 1975, AO 14, 743.

Kondrashova et al. : 1974, SP 34, 291.

Kostyk and Orlova: 1972, SP 26, 42.
Kostyk and Orlova: 1974, SP 36, 279.

Koutchmy et al.: 1973, Nature 246, 414.

Koutchmy and Magnant: $1973, A p J 186,671$.

Koutchmy and Koutchmy: 1974, AA 13, 295.

Koutchmy et al.: 1974, SP 35, 369.

Kozak and Korzhinskaya: 1973, SDB 6, 104.

Kozak and Kulchitsky: 1972, SDB 9, 73.

Kozak et al. : 1974, SDB 3, 98.

Krieger et al. : 1973, SP 29, 505.

Krieger et al.: 1974, Proc. Solar Wind Conf. Asilomar, Univ. Cal. Los Angeles.

Krishnaswamy and Stecher: 1974, ApJ 194, L153.

Krizhna et al. : 1974, ApJ 194, L153.

Kubicela: 1973, Proc. First European Astron. Meeting Athens, 1972, p. 123.

Kunasz et al. : 1973, AA 28, 15.

Kurokawa: 1975, SP (P).

Kurakowa et al.: 1974, SP 36, 69.

Kurucz: 1974, $S P$ 34, 17.

Kurucz: 1974, ApJ 188, L21.

Kurucz and Peytremann Avrett: 1974, Blanketed Model Atmospheres for Early Type Stars, Smithsonian Institute Press, Washington.

Lambert and Mallier: 1974, BAIC 25, 216.

Lamy and Koutchmy: 1975, $A A$ (P).

Lamy: 1974a, $A A$ 33, 191.

Lamy : $1974 \mathrm{~b}$ AA 35, 197.

Landi degl'Innocenti and Landi degl'Innocenti: 1972, SP 27, 319.

Landi degl'Innocenti and Landi degl'Innocenti: 1973a, SP 31, 299.

Landi degl'Innocenti and Noci: 1973b, SP 29, 287.

Landini and Monsignori-Fossi: 1975, AA (P).

Landman: 1973, SP 30, 371.

Landman, Orrall and Zane: 1973 in Giaspey Walker (ed.), Astron. Obs. with Television-Type Sensors, p. 255.

Lantos, Avignon: 1975, AA (P).

Leblanc et al.: 1973, SP 31, 343.

Leblanc et al.: 1974, SP 37, 215.

Lemaire and Skumanich: 1973, AA 22, 61 .

Le'Na et al. : 1974a, $A A$ 37, 75.

Le'Na et al. : 1974b, $A A$ 37, 81.

Lennard et al.: 1975, ApJ 197, 517.

Leroy: 1974, SP 36, 81.

Leroy and Ratier: 1974, Planets, Stars and Nebulae Studied with Photopolarimetry, Tucson, p. 762 .

Leroy and Trellis: 1974a, AA 35, 283.

Leroy and Trellis: 1974b, AA 35, 289.

Leroy et al.: 1973, SP 32, 131.

Levine: $1974 a, A p J ~ 190,447$.

Levine: $1974 b, A p J 190457$.

Levine and Altschuler: 1974, SP 36, 345.

Levy: 1974, AA 31, 451.

Liebenberg et al.: 1975, SP 40, 387.

Linsky: 1973, SP 28, 409.

Linsky et al. : 1975, $A p J(P)$.

Lites: 1973, SP 32, 283. 
Lites: 1974, $A A$ 36, 297.

Liu: 1974, ApJ 189, 359.

Liu and Skumanich: 1974, SP 38, 109.

Livingston and Mickey: 1972, SP 25, 267.

Livingston and Orral: 1974, SP 39, 301.

Livingston and White: 1974, SP 39, 289.

Livingston et al. : 1974, BAAS 6, 291.

Livshits and Badaliyan: 1974, AZ 51, 139.

Loughhead: 1969, SP 10, 71.

Loughhead: 1973, SP 29, 327.

Loughhead: 1974, SP 35, 55.

Loulergue and Nussbaumer: 1974, AA 34, 225.

Low and Nakagawa: 1975, ApJ 199, 237.

Lynch: 1974, BAAS 6, 291.

Lynch, Beckers and Dunn: 1973, SP 30, 63.

Lynch and Chapman: 1975, ApJ 197, 241.

Macris: 1974, Praktika of Athens Academy, 49, 215.

Magnan and Pecker: 1974, Highlights of Astronomy 3, 171.

Magnant-Crifo: 1973, SP 31, 94.

Magnant-Crifo: 1974, SP 39, 141.

Magnant-Crifo: 1975, SP 41, 109.

Malinovsky and Heroux: 1973, ApJ 181, 1009.

Mankin et al. : 1974, AA 31, 17.

Margrave: 1972, SP 27, 294.

Mariska and Withbroe: 1975, SP (P).

Marshall and Henderson: 1973, SP 33, 153.

Martin et al. : 1974, SP 37, 343.

Mason: $1975 \mathrm{a}, M N 170,651$.

Mason: $1975 \mathrm{~b}, M N 171,119$.

Matting-Nesis: 1974, SP 36, 3.

Mc Allister: 1974, SP 35, 3.

Mc Allister and Smith: 1975, SP 41, 3.

Mc Cabe : 1973, SP 30, 439.

Mc Intosh: 1972, RGSP 10,837.

Mc Whirter, Thonemann and Wilson: 1975, AA 40, 63.

Mehltretter: 1973, SP 30, 19.

Mein: 1971, SP 20, 3.

Mein and Blondel: 1972, SP 27, 489.

Mein and Mein: 1975, SP 40, 317 .

Mercier: 1973, SP 33, 177.

Michalitsanos: 1973, SP 30, 47.

Mickey: 1973, ApJ 181, L19.

Mickey and Orrall: 1974, Planets, Stors and Nebulae Studied with Photo-Polarimetry, Tucson p. 686.

Mickey, Orrall and Zane: 1972, Proc. SPIE 28, 217.

Milkey: 1975, ApJ 199, L131.

Milkey and Mihalas: 1973, SP 32, 361.

Milkey and Mihalas: 1974, ApJ 192, 769.

Milkey and Orrall: 1974, $A A$ 31, 179.

Milkey, Haasley and Beebe: 1973, ApJ 186, 1043.

Moe, Engvold and Beckers: $1975, S P 40,65$.

Mogilevsky et al. : 1973, SP 33, 169.

Molodensky: 1973, SP 28, 465.

Molodensky: 1974, SP 39, 393.

Moore: 1974, SP 37, 53.
Moore et al. : 1975, SSR (P).

Morozhenko: 1975, SP 42, 71.

Mount and Linsky: 1974a, SP 35, 259.

Mount and Linsky: 1974b, SP 35, 287.

Mount and Linskey: 1975, SP 41, 17.

Mount et al. : 1973, SP 32, 13.

Mouradian: 1974, CR, Serie B 279, 683.

Mukai and Mukai: 1973, PASJ 25, 481.

Mukai et al. : 1974, PASJ 26, 445.

Müller and Sauval: 1975, AA 39, 445.

Müller et al.: 1975, SP 41, 53.

Musman: 1974, SP 36, 313.

Mutschlecner: 1972, BAAS 4, 388.

Nakagawa: 1973, $A A$ 27, 95.

Nakagawa and Levine: 1974, ApJ 190, 441.

Nakagawa et al.: 1973, ApJ 184, 931.

Nakagawa and Priest: $1973, A p J 179,949$.

Nagasawa and Shirnizu: 1973, Tokyo Astron Obs. Repts 16, 545.

Neckel and Labs: 1973, IAU 54, 149.

Neupert and Pizzo: 1974, JGR 79, 3701.

Neupert et al.: 1972, BAAS 4, 388.

Neupert et al. : 1975, $U$.

Newkirk: 1972, Publ. Amer. Astronaut. Soc. Sci. Techn. Ser. 28, 47.

Newkirk: 1975, IAU 57.

Newkirk et al.: 1973, NCAR, Boulder.

Nicolaides and Sinanoglu: 1973, SP 29, 17.

Nishi: 1973, SP 33, 23.

Nishi: $1975, S P 42,37$.

Noci: 1973, SP 28, 403.

Noci and Rozelot: $1974, A A 30,81$.

Noyes and Hall: $1972 a, B A A S 4,389$.

Noyes and Hall: $1972 b, A p J 176, L 89$.

Noyes et al.: 1973, SP 28, 343.

Noyes et al.: 1975. IAU 68, 3

Nussbaumer and Storey: 1975, $A A(P)$.

Obashev and Minosyants: 1973, Soln. Dann. No. 2, 113.

Obashev, Milovanov and Kajdash: 1973, Trudy Astrofiz. Inst. Alma-Ata 23, 3.

Orrall and Speer: 1973, SP 29, 41.

Palsgard and Stenflo: 1970, SP 11, 155.

Parker: 1974, ApJ 190, 429.

Parker: 1974a, ApJ 189, 563.

Peraiah: 1973, SP 30, 29.

Perry and Altschuler: 1973, SP 28, 435.

Petford et al.: 1971, SP 19, 264.

Peytreymann: 1974, AA 33, 203.

Picat et al. : 1973, AA 24, 259.

Pick et al. : 1975, SP (P).

Piddington: 1973, SP 33, 363.

Pierce and Breckinridge: 1973, The Kitt Peak Table of Photographic Solar Spectrum Wavelengths

Pikel'ner: 1974, $A Z$ 51, 233.

Pinnington and Lutz: 1974, Can. J. Phys. 52, 1253.

Pinter: 1974, SP 35, 225. 
Plaskett: 1973, $M N$ 163, 183.

Pneuman: 1973, SP 28, 247.

Pope and Schoolman: 1975, SP 42, 47.

Poulain: 1974, SP 36, 339.

Priest and Pneuman: 1974, SP 34, 231.

Provost: 1975, SP 40, 257.

Punetha: 1974a, BAIC 25, 207.

Punetha: 1974b, BAIC 25, 212.

Querfeld: 1974, Planets, Stars and Nebulae Studied with Photo-Polarimetry, Tucson, p. 254.

Raadu and Kuperus: 1973, SP 28, 77.

Rachkowsky: 1972,IKAO 44, 64.

Ramsey: 1971, SP 21, 54.

Ramsay, Kobler and Mugridge: 1970, SP 12, 492.

Ratter: 1975, Nouvelle Revue d'Optique 6, 149.

Reeves et al.: 1974, ApJ 188, L27.

Rosenberg: 1975, SP (P).

Ross: 1973a, $A p J 180,599$.

Ross: $1973 \mathrm{~b}$, PASP 85, 537.

Ross and Aller: 1972, SP 25, 10.

Ross and Aller: 1974a, SP 36, 11.

Ross and Aller: 1974b, SP 35, 281.

Rottman: 1974, Am. Geophys. Union Meeting.

Roxburgh and Singer: 1975, SP 41, 241.

Rozelot and Noens: 1974, AA 32, 453.

Rozelot and Ratier: 1975, SP 40, 371.

Rusin: 1973, BAIC 24, 121.

Rusin and Rybansky: 1975, BAIC 26, 160.

Russell: 1974, Proc. Solar Wind Conf. Asilomar, Univ. Calif. Los Angeles.

Rutten: 1973, SP 28, 347.

Rutten et al. : 1974, SP 38, 321.

Sahal and Brechot: 1974a, $A A$ 32, 147.

Sahal and Brechot: $1974 \mathrm{~b}, A A \quad 36,355$.

Saito and Tandberg-Hanssen: 1973, SP 31, 105.

Samain et al.: 1975, $A A$ 39, 71.

Sandford et al. : 1973, ApJ 181, L15.

Sauval et al. : 1973, AA 27, 29.

Sauval et al.: 1975, AA 39, 445.

Sazanov: 1973, SA 16, 674.

Schatzman and Magnan: 1975, AA 38, 373.

Schmahl et al. : 1974, SP 39, 337.

Schmeleva and Syrovatskii: 1973, SP 33, 341.

Schmidtke: 1975, Space Res. XVI (P).

Sedlmayr: 1974, $A \boldsymbol{A}$ 31, 23.

Sentman and Shawhan: 1974, SP 35, 83.

Shine and Linsky: 1974, SP 37, 145.

Shine et al. : 1975, ApJ $\backslash(\mathrm{P})$.

Simon: 1974, Proc. 3rd Conf. Climatic Impact Ass. Program, p. 137.

Simon and Zirker: 1974, SP 35, 331.

Simon et al.: 1974, SP 39, 121.

Sinha and Pande: 1974, BAIC 25, 84.

Sivaraman: 1973a, SP 33, 319.

Sivaraman: 1973b, SP 33, 333.

Sivaraman: 1974, SP 36, 49.

Smith: 1974, ApJ 190, 481.

Smith and Tottlieb: 1974, SSR 16, 771.
Smith and Whaling: 1973, ApJ 183, 313.

Snider et al. : 1974, SP 36, 303.

Sorli and Engvold: 1972, Inst. Theor, Astrophys. Blindern, Oslo, 37,

Spruit: 1974, SP 34, 277.

Staude: 1972, SP 24, 255.

Stein and Leibacher: 1974, $A R A A$ 12, 407.

Stein and Schwartz: 1972, ApJ 177, 807.

Stellmacher and Koutchmy : 1974, $A A$ 35, 43.

Stenflo: 1973a, $A O 12,805$.

Stenflo: 1973b, SP 32, 41.

Stenflo: 1974, SP 37, 31.

Stettler et al.: 1972, AA 20, 309.

Stix and Wöhl: 1974, SP 37, 63.

Streete and Tandberg-Hansen: 1974, AA 33, 385.

Summers: $1974, M N 169,663$.

Svalgaard et al. : 1974, SP 37, 157.

Svensson, Eckberg and Edlen: 1974, SP 34, 173.

Sýkora: 1975, BAIC 27, (P).

Sýkora et al. : 1975, SP (P).

Ta and Chunli: 1973, $A O 12,2828$.

Tallant: 1970, SP 11, 263.

Tanaka and Enome: 1975, SP 40, 123.

Tetruashvili: 1975, SP 39, 387.

Teske: $1974 a, S P$ 39, 363.

Teske: $1974 b, S P$ 39, 79.

Thekaekara: 1974, $A O$ 13, 518.

Thomas: 1972, SP 27, 303.

Thomas et al.: 1972, $A L 12,31$.

Timothy et al.: 1975, SP 42, 135.

Title: $1973, S P, 33,521$.

Title: 1974, $S P$ 38, 523.

Tjotte and Tjötta: 1974, $A A$ 30, 349.

Tousey et al.: 1973, SP 33, 265.

Tousey et al. : 1974, NRL Report 7788.

Tousey et al. : 1975, IAU 68, 140.

Tousey et al:: 1975 , Atlas of the Solar UV Spectrum between 2226 and 2992 A.

Troyen: 1973, $S D B 4,89$.

Tucker: 1973, $A p J 186,285$.

Turon: 1975, SP 41, 271.

Uchida and Kaburaki: 1974, SP 35, 451.

Ulmschneider: 1974, SP 39, 327.

Ulmschneider and Kalkofen: 1973, SP 28, 3.

Ulrich: 1970, ApJ 162, 993.

Vaiana et al. : 1973, ApJ 185, L47.

Van Dessel: 1974, SP 38, 351.

Van Dessel et al. : 1973, SP 33, 375.

Van Dessel et al. : 1974, SP 38, 351.

Van Rensbergen et al.: 1975, SP 40, 303.

Vardavas and Cram: 1974, SP 38, 367

Vasilév: 1973, $S A$ 16, 875.

Vernazza et al.: 1975, ApJ 199, L123.

Vernazza, Avrett and Loeser: 1975, ApJ (P).

Vilkoviskii: 1973, SA 16, 918.

Wagner: 1975, ApJ 198, L141.

Waldmeier: 1974, AMZ 330. 
Waldmeier: 1975a, SP 40, 351.

Waldmeier: 1975b, $A M Z 331$.

Waldmeier and Weber: 1974, $A M Z 329$.

Walker, Rugge and Weiss: 1974, $A p J 192,169$.

Weber and Rosenberg: 1974, SP 37, 409.

Wentzel: 1974, SP 39, 129.

Wiehr: 1971, SP, 18, 226.

Wiehr: 1975, $A A$ 38, 303.

Wiehr and Rossbach: 1974, SP 35, 351.

Wilcox and Svalgaard: 1974, SP 34, 461.

Wilson and Guidry: 1974, $M N$ 166, 219.

Withbroe: 1970, SP 11, 42.

Withbroe: 1971. Menzel Symposium, Nat.Bur.Standards, Spec. Publ. 353, p. 127.
Withbroe and Gurman: 1973, ApJ 183, 279.

Withbroe et al. ApJ (P).

Wittman: 1974, SP 35, 11.

Wöhl: 1974, $A A$ 34, 41.

Wöhl: 1975, $A A$ 40, 343.

Wolff: $1972, A p J 177,487$.

Wolff: 1973, SP 32, 31 .

Worden: 1975, Thesis Univ. Arizona.

Worral: 1973, AA 29, 37.

Zhugzhda: 1972, SP 25, 329.

Zhugzhda: 1973, $A L 15,119$.

Zirin: 1974, SP 38, 91.

Zirin: 1975, ApJ 199, L63.

R. G. GIOVANELLI

President of the Commission 